\title{
Group theoretic interpretations of Askey's scheme of hypergeometric orthogonal polynomials
}

\author{
Tom H. Koornwinder* \\ Centre for Math. and Computer Science, Amsterdam and Math. Institute, University of Leiden
}

\section{Introduction}

The concept of classical orthogonal polynomials changed quite drastically during the last decade (cf. Andrews and Askey [1]). The present consensus about what should be included in this class can be neatly summarized in the Askey scheme of hypergeometric orthogonal polynomials, cf. Askey and Wilson [4, Appendix], Labelle [30] and §5, Table 3 in the present paper. A remarkable aspect of this scheme are the arrows indicating limit transitions between the various families of orthogonal polynomials, starting at the top with the Wilson and Racah polynomials and ending at the bottom with the Hermite polynomials.

Almost all members of the Askey scheme have group theoreretic interpretations. The present paper gives a partial survey of these connections with group theory, but, at the same time, it tries to illustrate a philosophy and a research program. The philosophy consists of the following:

1. Almost any identity for special functions in, say, the Bateman project [14], [16] has a deeper meaning in (i) the harmonic analysis of orthogonal systems of special functions and (ii) some group theoretic context.

2. The Askey scheme is a subgraph of a much bigger scheme of families of unitary integral transforms with hypergeometric kernels. From this point of view the selection criterium that these kernels should be of polynomial nature is quite arbitrary.

3. The integral transforms occurring in the (extended) Askey scheme can be grouped together in triples (in many different ways) such that the three transforms in a triple, when performed successively, yield identity. The limit transitions in the scheme often extend to limit transitions for these triples.

The research program naturally follows from this philosophy. Its main task is to make the three above items concrete and to fill them in as completely as possible. Partly this can be done by a search through the literature, partly new research is needed. However, there are also related problems of more fundamental nature, for instance the following one:

The spectral theory of second order difference operators on $\mathbb{Z}_{+}$and of second order differential operators on an interval (possibly with singularities at the end points) is quite well understood nowadays. The first theory is closely related to the general theory of orthogonal polynomials and the second theory to the integral transform theory with respect to the eigenfunctions of a second order differential operator, cf. for instance Trimèche [35]. Classical orthogonal polynomials $p_{n}(x)$ are not only eigenfunctions of a difference operator in $n$ but also of a second order diferential or difference operator in $x$. "Classical" eigenfunctions $\phi_{\lambda}(t)$ of a second order differential operator in $t$ are also eigenfunctions of a second order differential or difference operator in $\lambda$. What would be helpful, but is still missing, is a general spectral theory of second order difference operators, not just on $\mathbb{Z}_{+}$, but also on $\mathbb{C}$, with the difference operator being analytic, possibly with singularities. Recent work by Ruijsenaars [32], related to completely integrable systems in relativistic quantum mechanics, also shows the need for such a theory.

The guiding formula for this paper is an identity yielding Wilson polynomials as Jacobi function transforms of Jacobi polynomials, cf. Koornwinder [27, \$9], [29] and formula (4.20) in the present paper. We gradually approach this identity in sections 2,3 and 4 , starting with formulas which contain a differential operator $p_{k}(i d / d t)$, where $p_{k}$ is an orthogonal polynomial (based on joint work with E. Badertscher [6]). At the end of $\$ 4$ we have already seen some illustration of the

* Mail address: CWI, P.O.Box 4079, 1009 AB Amsterdam, The Netherlands 
philosophy developed above and we are now ready to discuss in $\$ 5$ the Askey scheme and its possible extensions. Until here, not much group theory did enter, except some in §3. In section 6 we discuss some fundamental ways to construct orthogonal systems from group representations. The rest of the paper gives examples of these constructions which yield orthogonal systems of hypergeometric functions fitting into the (extended) Askey scheme: at the end of $\S 6$ for $S U(2)$ and the Euclidean motion group $I_{0}\left(\mathbb{R}^{2}\right)$, in $\S 7$ for the discrete series representations of $S L(2, \mathbb{R})$ (following Basu and Wolf [9]), in $\$ 8$ the Hahn and Racah polynomials and, finally, in $\S 9$ the Wilson polynomials, where the above-mentioned identity finds its proper setting.

The group theoretic interpretations discussed in this paper only use $S U(2), S L(2, \mathbb{R}), I_{0}\left(\mathbb{R}^{2}\right)$, the rotation groups and the generalized Lorentz groups. By lack of space and time many other groups relevant for the Askey scheme are not discussed here, for instance the Heisenberg group and the symmetric group. We also avoided discussion of $q$-Wilson polynomials, mainly because known group theoretic interpretations for orthogonal polynomials of $q$-hypergeometric type are restricted to Chevalley groups and homogeneous trees.

The analysis in this paper remains at the formal level. The convergence of series and integrals, the precise sense in which one family of the Askey scheme approximates another family and the interpretation of inner products of distributions are not considerd here. However, the author is preparing a paper, where these questions will be dealt with.

\section{Continuous Hahn polynomials: Playing around with some remarkable formulas}

Consider Bessel functions

$$
J_{k}(t):=\frac{(1 / 2 t)^{k}}{k !}{ }_{0} F_{1}\left(k+1 ;-1 / 4 t^{2}\right)
$$

of order $k=0,1,2, \cdots$ with integral representation

$$
J_{k}(t)=\pi^{-1} i^{-k} \int_{0}^{\pi} e^{i t \cos \psi} \cos (k \psi) d \psi
$$

(cf. [14, Vol.2, 7.3(2)]). Substitute the expression for the Chebyshev polynomial

$$
T_{k}(\cos \psi):=\cos (k \psi)
$$

in (2.2) and make the change of variable $x:=\cos \psi$ in the integral. Then we obtain

$$
J_{k}(t)=\pi^{-1} i^{-k} \int_{-1}^{1} e^{i t x} T_{k}(x)\left(1-x^{2}\right)^{-1 / 2} d x .
$$

In particular, for $k=0$ :

$$
J_{0}(t)=\pi^{-1} \int_{-1}^{1} e^{i t x}\left(1-x^{2}\right)^{-1 / 2} d x
$$

Let us now build from the polynomial $T_{k}$ the differential operator with constant coefficients $T_{k}(-i d / d t)$. If we let this operate on (2.5) then we obtain

$$
T_{k}(-i d / d t) J_{0}(t)=\pi^{-1} \int_{-1}^{1} e^{i t x} T_{k}(x)\left(1-x^{2}\right)^{-1 / 2} d x .
$$

Hence, in view of (2.4), we get the curious formula

$$
T_{k}(-i d / d t) J_{0}(t)=i^{k} J_{k}(t) \text {. }
$$

Observe that, by (2.5), $J_{0}$ is the Fourier transform of the orthogonality measure for which the polynomials $T_{k}$ are orthogonal.

We try to play the same game with the associated Legendre functions 


$$
\begin{aligned}
& P_{i \lambda-1 / 2}^{k}(\operatorname{ch} t):=\frac{2^{-k} \Gamma(i \lambda+k+1 / 2)}{k ! \Gamma(i \lambda-k+1 / 2)}(\operatorname{sh} t)^{k} \\
& \times_{2} F_{1}\left(i \lambda+k+1 / 2,-i \lambda+k+1 / 2 ; k+1 ;-(\operatorname{sh}(1 / 2 t))^{2}\right),
\end{aligned}
$$

where $t \in \mathbb{R}, \lambda \in \mathbb{C}, k \in \mathbb{Z}_{+}$, with integral representation

$$
P_{i \lambda-1 / 2}^{k}(\operatorname{ch} t)=\frac{(i \lambda+1 / 2)_{k}}{\pi k !} \int_{0}^{\pi}(\operatorname{ch} t+\operatorname{sh} t \cos \psi)^{i \lambda-1 / 2} \cos (k \psi) d \psi
$$

(cf. $[14,3.6(1), 3.7(14)])$. Make again the substitution (2.3) in (2.8) and make the change of variable th $y:=-\cos \psi$ in the integral. Then (2.8) becomes

$$
P_{i \lambda-1 / 2}^{k}(\operatorname{ch} t)=\frac{(-1)^{k}(i \lambda+1 / 2)_{k}}{\pi k !} \int_{-\infty}^{\infty}(\operatorname{ch}(t-y))^{i \lambda-1 / 2} T_{k}(\operatorname{th} y)(\operatorname{ch} y)^{-i \lambda-1 / 2} d y,
$$

which we recognize as a convolution product on $\mathbb{R}$. In particular, for the Legendre function $P_{i \lambda-1 / 2}:=P_{i \lambda-1 / 2}^{0}$ we obtain

$$
P_{i \lambda-1 / 2}(\operatorname{ch} t)=\pi^{-1} \int_{-\infty}^{\infty}(\operatorname{ch}(t-y))^{i \lambda-1 / 2}(\operatorname{ch} y)^{-i \lambda-1 / 2} d y .
$$

Hence

$$
(i d / d t)^{k} P_{i \lambda-1 / 2}(\operatorname{ch} t)=\pi^{-1} \int_{-\infty}^{\infty}(\operatorname{ch}(t-y))^{i \lambda-1 / 2}(i d / d y)^{k}(\operatorname{ch} y)^{-i \lambda-1 / 2} d y .
$$

One easily proves by induction with respect to $k$ that

$$
(\operatorname{ch} y)^{i \lambda+1 / 2}(i d / d y)^{k}(\operatorname{ch} y)^{-i \lambda-1 / 2}=(-i)^{k}(i \lambda+1 / 2)_{k}(\text { th } y)^{k}+\text { lower degree in th } y \text {. }
$$

It follows that, for each $\lambda \in \mathbb{C}$ with $-i \lambda-1 / 2 \notin \mathbb{Z}_{+}$there are unique polynomials $p_{k}$ of degree $k$ such that

$$
p_{k}(i d / d y)(\operatorname{ch} y)^{-i \lambda-1 / 2}=\text { const. } T_{k}(\text { th } y)(\operatorname{ch} y)^{-i \lambda-1 / 2} .
$$

For these polynomials we conclude:

$$
p_{k}(i d / d t) P_{i \lambda-1 / 2}(\mathrm{ch} t)=\text { const. } P_{i \lambda-1 / 2}^{k}(\operatorname{ch} t) \text {. }
$$

We will show that the polynomials $p_{k}$ are orthogonal on $\mathbb{R}$ with respect to a weight function $w$ which can be explicitly determined. Observe from (2.7) that the function $t \mapsto P_{i \lambda-1 / 2}^{k}(\operatorname{ch} t)$ has a zero of order $k$ at 0 . Let $l<k$. Then

$$
\begin{aligned}
0 & =\text { const. }\left.(i d / d t)^{l} P_{i \lambda-1 / 2}^{k}(\operatorname{ch} t)\right|_{t=0} \\
& =\left.(i d / d t)^{l} p_{k}(i d / d t) P_{i \lambda-1 / 2}(\operatorname{ch} t)\right|_{t=0} \\
& =(2 \pi)^{-1} \int_{-\infty}^{\infty} \mu^{l} p_{k}(\mu) w(\mu) d \mu,
\end{aligned}
$$

where

$$
\begin{aligned}
w(\mu) & =\int_{-\infty}^{\infty} P_{i \lambda-1 / 2}(\operatorname{ch} t) e^{i \mu t} d t \\
& =(\pi)^{-1}\left[\int_{-\infty}^{\infty}(\operatorname{ch} t)^{i \lambda-1 / 2} e^{i \mu t} d t\right]\left[\int_{-\infty}^{\infty}(\operatorname{ch} t)^{-i \lambda-1 / 2} e^{i \mu t} d t\right)
\end{aligned}
$$

by $(2.10)$. Hence, by $[14,1.5(26)]$ we obtain

$$
w(\mu)=\text { const. } \Gamma(1 / 2(i \mu+i \lambda+1 / 2)) \Gamma(1 / 2(-i \mu+i \lambda+1 / 2))
$$




$$
\times \Gamma(1 / 2(i \mu-i \lambda+1 / 2)) \Gamma(1 / 2(-i \mu-i \lambda+1 / 2)) .
$$

Here, for convergence of the integrals, we require that $|\operatorname{Im} \lambda|<1 / 2$. It follows that the polynomials $p_{k}$ are orthogonal on $\mathbb{R}$ with respect to the weight function $w$. In particular, if $\lambda \in \mathbb{R} \cup(-1 / 2 i, 1 / 2 i)$ then this weight function is positive. The polynomials orthogonal with respect to the weight function given by (2.13) are particular cases of the continuous symmetric Hahn polynomials

$$
p_{k}(x ; a, b):=i_{3}^{k} F_{2}\left(\begin{array}{c}
-k, k+2 a+2 b-1, a-i x \\
a+b, 2 a
\end{array} \mid 1\right),
$$

which are orthogonal on $\mathbb{R}$ with respect to the weight function $x \mapsto|\Gamma(a+i x) \Gamma(b+i x)|^{2}(a, b>0$ or $b=\bar{a}, \operatorname{Re} a>0$ ), cf. Askey and Wilson [3]. From this reference we find that our polynomials $p_{k}$ can be written as

$$
\begin{aligned}
p_{k}(\mu) & =\text { const. } p_{k}(1 / 2 \mu ; 1 / 4+1 / 2 i \lambda, 1 / 4-1 / 2 i \lambda) \\
& =\text { const. } i^{k} F_{2}\left(\begin{array}{c|c}
-k, k, 1 / 4+1 / 2 i \lambda-1 / 2 i \mu & 1 \\
1 / 2,1 / 2+i \lambda & 1
\end{array} .\right.
\end{aligned}
$$

So, similarly as with (2.6), the function $t \mapsto P_{i \lambda-1 / 2}(\operatorname{ch} t)$ in (2.12) is the Fourier transform of the orthogonality measure for which the polynomials $p_{k}$ in (2.12) are orthogonal.

We now have three formulas of the same structure:

$$
\begin{aligned}
& J_{k}(t)=\text { const. } T_{k}(i d / d t) J_{0}(t), \\
& P_{i \lambda-1 / 2}^{k}(\operatorname{ch} t)=\text { const. } p_{k}(1 / 2 i d / d t ; 1 / 4+1 / 2 i \lambda, 1 / 4-1 / 2 i \lambda) P_{i \lambda-1 / 2}(\operatorname{ch} t), \\
& P_{n}^{k}(\cos \theta)=\text { const. } Q_{k}(1 / 2 n+1 / 2 i d / d \theta ;-1 / 2,-1 / 2, n) P_{n}(\cos \theta) .
\end{aligned}
$$

Here (2.17) is obtained from (2.12) and (2.15), while (2.18) is obtained from (2.17) by taking $i \lambda-1 / 2$ as an integer $\geqslant k$ and by taking $t$ purely imaginary. Then $P_{n}$ in (2.18) becomes the Legendre polynomial while

$$
Q_{k}(1 / 2 n+1 / 2 m ;-1 / 2,-1 / 2, n):={ }_{3} F_{2}\left(\begin{array}{c}
-k, k_{1 / 2},-1 / 2 n-1 / 2 m \\
\mid 1
\end{array}\right)
$$

is a special Hahn polynomial, orthogonal on $\{-n,-n+2, \ldots, n\}$ with respect to the weights

$$
w_{m}=\frac{(1 / 2)_{1 / 2 n+1 / m}(1 / 2)_{1 / 2 n-1 / 2 m}}{(1 / 2 n+1 / 2 m) !(1 / 2 n-1 / 2 m) !}
$$

cf. Karlin and McGregor [22] except for the standardized notation, which is given in Askey and Wilson [4]. Note that the weights $w_{m}$ are the Fourier coefficients of $P_{n}(\cos \theta)$ :

$$
P_{n}(\cos \theta)=\sum_{m=-n,-n+2, \ldots, n} w_{m} e^{i m \theta},
$$

cf. Szegö [34, (4.9.19)].

Formula (2.16) is a limit case of both (2.17) and (2.18): Replace $t$ (or $\theta)$ by $\lambda^{-1} t\left(\right.$ or $\left.n^{-1} \theta\right)$ and let $\lambda \rightarrow \infty$ (or $n \rightarrow \infty)$.

\section{Orthogonal polynomials of argument $i d / d t$. A group theoretic interpretation}

Corresponding to the formulas (2.16), (2.17), (2.18), respectively, we consider the Lie groups $G=$ $I_{0}\left(\mathbb{R}^{2}\right), S O_{0}(1,2)$ and $S O(3)$. Here $I_{0}\left(\mathbb{R}^{2}\right)$ is the group of orientation preserving Euclidean motions of $\mathbb{R}^{2}, S O_{0}(1,2)$ is the connected component of the group of linear transformations of $\mathbb{R}^{3}$ which leave invariant the quadratic form $-x^{2}+y^{2}+z^{2}$ and $S O$ (3) the group of rotations around the origin in $\mathbb{R}^{3}$. These groups $G$ have natural transtive actions on certain spaces $\Omega$, i.e., respectively, on $\mathbb{R}^{2}$, on a sheet of the hyperboloid $-x^{2}+y^{2}+z^{2}=1$ and on the unit sphere $S^{2}$ in $\mathbb{R}^{3}$. In all cases the subgroup $K$ of $G$ leaving some point of $\Omega$ fixed is isomorphic to $S O(2)$, the group of rotations of the circle. So $\Omega$ can be identified with the homogeneous space $G / K$.

By a unitary representation $\pi$ of $G$ on a Hilbert space $\mathscr{T}(C=\mathscr{F}(\pi)$ ) we mean a mapping $\pi$ of $G$ into the group of unitary transformations of $\mathcal{H}$ which is a group. homomorphism (i.e. $\pi\left(g_{1} g_{2}\right)=\pi\left(g_{1}\right) \pi\left(g_{2}\right)$ for all $g_{1}, g_{2} \in G$ ) and which is strongly continuous (i.e. for all $v \in \mathcal{H}$ the 
mapping $g \mapsto \pi(g) v: G \rightarrow \mathcal{T}$ is continuous).

The representation $\pi$ of $G$ is called irreducible if the only closed $G$-invariant subspaces of $\mathcal{F}(\pi)$ are $S(\pi)$ and $\{0\}$. The set of all irreducible unitary representations of $G$ (more precisely, of all equivalence classes of irreducible unitary representations of $G$ ) is denoted by $G$.

We can identify the group $K$ with the group $S O(2)$ of matrices

$$
u_{\theta}:=\left(\begin{array}{rr}
\cos \theta & \sin \theta \\
-\sin \theta & \cos \theta
\end{array}\right), \quad \theta \in \mathbb{R} .
$$

It can be shown that the three pairs $(G, K)$ as considered above share the following property: For each $\pi \in \hat{G}$ there is a subset $I$ of $\mathbb{Z}$ and an orihcinormal basis $\left\{e_{k}\right\}_{k \in I}$ of $\mathcal{F}(\pi)$ such that

$$
\pi\left(u_{\theta}\right) e_{k}=e^{i k \theta} e_{k}, \quad k \in I, \theta \in \mathbb{R} .
$$

Otherwise stated: For each $\pi \in \hat{G}$ the restriction of $\pi$ to $K$ is multiplicity free.

Definition. Let $\pi \in \hat{G}$ and denote the inner product on $\mathscr{T}(\pi)$ by $(.,$.$) . Suppose that e_{0}$ occurs in $\mathscr{K}$. Then

$$
\phi(g):=\left(\pi(g) e_{0}, e_{0}\right), \quad g \in G,
$$

denotes the spherical function $\phi$ for the pair $(G, K)$ and the representation $\pi$. If, for some $k \in \mathbb{Z}, e_{k}$ also occurs in $\mathcal{H}$ then

$$
\phi^{k}(g):=\left(\pi(g) e_{0}, e_{k}\right), \quad g \in G,
$$

denotes an associated spherical function for $(G, K)$ and $\pi$.

Note that $\phi$ and $\phi^{k}$ are right invariant with respect to $K$, so they can be considered as functions on $G / K$. Moreover, the spherical function $\phi$ is left invariant with respect to $K$.

For each group $G$ as considered above there is a one-parameter subgroup $A=\left\{a_{t}\right\}_{t \in \mathbb{R}}$ of $G$ such that we have the Cartan decomposition $G=K A K$, i.e., each $g \in G$ can be written as $g=k_{1} a_{t} k_{2}$ for some $k_{1}, k_{2} \in K$ and $t \in \mathbb{R}$. Now it can be shown that, after possibly rescaling of $\left\{a_{t}\right\}$, the associated spherical functions $\phi^{k}$, when restricted to $A$, only change by a constant factor if $k$ is replaced by $-k$ and that, up to a constant factor, $\phi^{k}\left(a_{t}\right)$ with $k \geqslant 0$ equals

$$
\begin{aligned}
& J_{k}(\lambda t) \text { for some } \lambda \in \mathbb{R} \text { or } \\
& P_{i \lambda-1 / 2}^{k}(\operatorname{ch} t) \text { for some } \lambda \in \mathbb{R} \cup[-1 / 2 i, 1 / 2 i] \text { or } \\
& P_{n}^{k}(\cos t) \text { for some } n \in \mathbb{Z}_{+},
\end{aligned}
$$

according to whether $G=I_{0}\left(\mathbb{R}^{2}\right), S O_{0}(1,2)$ or $S O(3)$. These are essentially the left hand sides of (2.16), (2.17) and (2.18). So, roughly stated, we have

$$
\phi^{k}\left(a_{t}\right)=\text { const. } p_{k}(i d / d t) \phi\left(a_{t}\right) \text {, }
$$

where $p_{k}$ is an orthogonal polynomial with respect to a measure which is the Fourier transform of $t \mapsto \phi\left(a_{t}\right)$.

In Badertscher and Koornwinder [7] it is shown that, with $e_{0}, e_{k}$ and $p_{k}$ as above, we have

$$
e_{k}=\text { const. }\left.p_{k}(i d / d t) \pi\left(a_{t}\right) e_{0}\right|_{t=0}
$$

(the function $t \mapsto \pi\left(a_{t}\right) e_{0}: \mathbb{R} \rightarrow \mathcal{S C}$ is $C^{\infty}$ ). Hence, in view of (3.1) and (3.2), we get

$$
\phi^{k}(g)=\text { const. }\left.p_{k}(i d / d t) \phi\left(a_{-t} g\right)\right|_{t=0}, \quad g \in G,
$$

which implies (3.3). Formula (3.50) provides us with an explicit right-invariant differential operator on $G$ which sends the spherical function $\phi$ to the associated spherical function $\phi^{k}$.

In the case that $G$ is an arbitrary connected semisimple Lie group with finite center and $K$ a 
maximal compact subgroup, Helgason [20, section 4] has a definition of associated spherical function (there called generalized spherical function) which generalizes our definition (3.2) and it is proved in [20, Theorem 4.1] that, under certain conditions for the corresponding representation, there exists a right-invariant differential operator on $G$ which sends the spherical function to the associated spherical function. However, Helgason does not specify this differential operator in any sense.

\section{Integral transforms mapping systems of orthogonal polynomials onto each other}

It follows from (2.11) and (2.15) that

$$
T_{k}(\operatorname{th} y)(\operatorname{ch} y)^{-i \lambda-1 / 2}=\text { const. } p_{k}(1 / 2 i d / d y ; 1 / 4+1 / 2 i \lambda, 1 / 4-1 / 2 i \lambda)(\operatorname{ch} y)^{-i \lambda-1 / 2} .
$$

Take Fourier transforms on both sides and use [14, 1.5(26)]. It follows that

$$
\begin{aligned}
& \int_{-\infty}^{\infty} T_{k}(\operatorname{th} y)(\operatorname{ch} y)^{-i \lambda-1 / 2} e^{i \mu y} d y=\text { const. } \Gamma(1 / 2(i \mu+i \lambda+1 / 2)) \\
& \times \Gamma(1 / 2(-i \mu+i \lambda+1 / 2)) p_{k}(1 / 2 \mu ; 1 / 4+1 / 2 i \lambda, 1 / 4-1 / 2 i \lambda) .
\end{aligned}
$$

It is clear from the orthogonality relations for the Chebyshev and continuous symmetric Hahn polynomials that the functions

$$
y \mapsto T_{k}(\operatorname{th} y)(\operatorname{ch} y)^{-i \lambda-1 / 2}, \quad k=0,1,2, \cdots
$$

form an orthogonal basis of $L^{2}(\mathbb{R}, d y)$ and the functions

$$
\mu \mapsto \Gamma(1 / 2(i \mu+i \lambda+1 / 2)) \Gamma(1 / 2(-i \mu+i \lambda+1 / 2)) p_{k}(1 / 2 \mu ; 1 / 4+1 / 2 i \lambda, 1 / 4-1 / 2 i \lambda), \quad k=0,1,2, \cdots
$$

an orthogonal basis of $L^{2}(\mathbb{R}, d \mu)$ and that the Fourier transform maps the first basis onto the second basis.

More generally there is an identity of type (4.2) for Gegenbauer polynomials

$$
C_{k}^{\alpha}(x):=\frac{(2 \alpha)_{k}}{k !}{ }_{2} F_{1}(-k, k+2 \alpha ; \alpha+1 / 2 ; 1 / 2-1 / 2 x),
$$

which are orthogonal on $(-1,1)$ with respect to the weight function $x \mapsto\left(1-x^{2}\right)^{\alpha-1 / 2}$. We have (cf. [8])

$$
\begin{aligned}
& \int_{-\infty}^{\infty}\left[C_{k}^{\alpha}(\operatorname{th} y)(\operatorname{ch} y)^{-i \lambda-\alpha-1 / 2}\right] e^{i \mu y} d y=\text { const. } \Gamma(1 / 2(i \mu+i \lambda+\alpha+1 / 2)) \\
& \times \Gamma(1 / 2(-i \mu+i \lambda+\alpha+1 / 2)) p_{k}(1 / 2 \mu ; 1 / 4+1 / 2 \alpha+1 / 2 i \lambda, 1 / 4+1 / 2 \alpha-1 / 2 i \lambda) .
\end{aligned}
$$

Here the functions of $y$ occurring in square brackets at the left hand side form an orthogonal basis of $L^{2}(\mathbb{R}, d y)$, while the functions of $\mu$ at the right hand side form an orthogonal basis of $L^{2}(\mathbb{R}, d \mu)$, and the two bases are mapped onto each other by the Fourier transform.

A much more elementary result of this type is the well-known formula

$$
(2 \pi)^{-1 / 2} \int_{-\infty}^{\infty} H_{k}(y) e^{-1 / 2 y^{2}} e^{i \mu y} d y=i^{-k} H_{k}(\mu) e^{-1 / 2 \mu^{2}},
$$

where $H_{k}$ is the Hermite polynomial, orthogonal on $\mathbb{R}$ with respect to the weight function $y \mapsto e^{-y^{2}}$. Actually, (4.5) can be obtained as a limit case of (4.4). Just replace $y$ by $\alpha^{-1 / 2} y$ and $\mu$ by $\alpha^{1 / 2} \mu$ in (4.4) and let $\alpha \rightarrow \infty$. Schematically we display the result in Table 1 below.

There is a further generalization of (4.4) which takes the Jacobi function transform of Jacobi polynomials rather than the Fourier transform of Gegenbauer polynomials. Jacobi polynomials are given by

$$
P_{n}^{(\alpha, \beta)}(x):=\frac{(\alpha+1)_{n}}{n !}{ }_{2} F_{1}(-n, n+\alpha+\beta+1 ; \alpha+1 ; 1 / 2(1-x)), \quad n=0,1,2, \cdots
$$

For $\dot{\alpha, \beta}>-1$ these are orthogonal polynomials on $(-1,1)$ with respect to the weight function 
Gegenbauer
polynomial

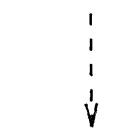

Hermite polynomial

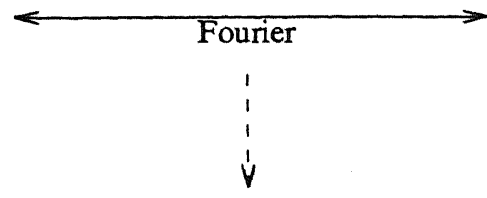

Fourier

continuous symmetric

Hahn polynomial

Table 1

$$
\Delta_{\alpha, \beta}(t):=(2 \operatorname{sh} t)^{2 \alpha+1}(2 \operatorname{ch} t)^{2 \beta+1} .
$$

and

$$
\phi_{\lambda}^{(\alpha, \beta)}(0)=1
$$

and $\phi_{\lambda}^{(\alpha, \beta)}$ is uniquely defined by these properties. Let

$$
c_{\alpha, \beta}(\lambda):=\frac{2^{\alpha+\beta+1-i \lambda} \Gamma(\alpha+1) \Gamma(i \lambda)}{\Gamma(1 / 2(i \lambda+\alpha+\beta+1)) \Gamma(1 / 2(i \lambda+\alpha-\beta+1))} .
$$

Then, for $\alpha>-1, \beta \in[-\alpha-1, \alpha+1] \cup i \mathbb{R}$ we have the integral transform pair

$$
\left.\begin{array}{l}
g(\lambda)=\int_{0}^{\infty} f(t) \phi_{\lambda}^{(\alpha, \beta)}(t) \Delta_{\alpha, \beta}(t) d t \\
f(t)=(2 \pi)^{-1} \int_{0}^{\infty} g(\lambda) \phi_{\lambda}^{(\alpha, \beta)}(t)\left|c_{\alpha, \beta}(\lambda)\right|^{-2} d \lambda
\end{array}\right\}
$$

This establishes a 1-1 correspondence between the space of even $C^{\infty}$-functions on $\mathbb{R}$ with compact support and its image under the Fourier-cosine transform, as characterized by the classical PaleyWiener theorem. The mapping $f \mapsto g$ (which we call the Jacobi function transform) extends to an isometry of Hilbert spaces between $L^{2}\left(\mathbb{R}_{+}, \Delta_{\alpha, \beta}(t) d t\right)$ and $L^{2}\left(\mathbb{R}_{+},(2 \pi)^{-1}\left|c_{\alpha, \beta}(\lambda)\right|^{-2} d \lambda\right)$. In the case that $\alpha>-1$ and $|\beta|>\alpha+1,(4.12)$ remains valid except that we have to add to the right hand side of the second formula a term

$$
\sum_{\lambda \in D_{\alpha, \beta}} g(\lambda) d_{\alpha, \beta}(\lambda)
$$

where the finite subset $D_{\alpha, \beta}$ of $i \mathbb{R}_{+}$and the weights $d_{\alpha, \beta}(\lambda)$ can be explicitly given. See [24], [27] and [19, Appendix 1] for further details about Jacobi functions.

Noteworthy special cases of Jacobi functions are

$$
\begin{aligned}
& \phi_{\lambda}^{(-1 / 2,-1 / 2)}(t)=\cos (\lambda t), \\
& \phi_{\lambda}^{(1 / 2,-1 / 2)}(t)=\frac{\sin (\lambda t)}{\lambda \operatorname{sh} t}, \\
& \phi_{\lambda}^{(0,-1 / 2)}(t)=P_{i \lambda-1 / 2}(\operatorname{ch} t) .
\end{aligned}
$$


Thus, for $\beta=-1 / 2,(4.12)$ reduces to the Fourier-cosine transform pair if $\alpha=-1 / 2$, to the Fourier-sine transform pair if $\alpha=1 / 2$ and to the Mehler-Fock transform pair if $\alpha=0$.

As a generalization of (4.4) we now want to evaluate

$$
\begin{aligned}
& \int_{0}^{\infty}\left[(\operatorname{th} t)^{\alpha+1 / 2}(\operatorname{ch} t)^{-\delta-i \mu-1} P_{n}^{(\alpha, \delta)}\left(1-2 \operatorname{th}^{2} t\right)\right] \\
& \times\left[\frac{\Gamma(1 / 2(\alpha+\beta+1+i \lambda)) \Gamma(1 / 2(\alpha-\beta+1+i \lambda))}{\Gamma(i \lambda)}(\operatorname{sh} t)^{\alpha+1 / 2}(\operatorname{ch} t)^{\beta+1 / 2} \phi_{\lambda}^{(\alpha, \beta)}(t)\right] d t
\end{aligned}
$$

where $\beta, \lambda, \mu \in \mathbb{R}$ and $\alpha, \delta>-1$. Indeed, (4.16) reduces to the left hand side of (4.4), up to a constant factor, if $\beta=-1 / 2, \alpha= \pm 1 / 2$ and $\delta$ is replaced by $\alpha-1 / 2$. (Use (4.13),(4.14) and the quadratic transformations for Jacobi polynomials.)

Let moreover $|\beta|>\alpha+1$ in (4.16). In view of the orthogonality relations for Jacobi polynomials and the isometry property of the Jacobi function transform, the functions of $\lambda$ defined by (4.16) for $n=0,1,2, \cdots$ will form an orthogonal basis of $L^{2}\left(\mathbb{R}_{+}\right)$. One can evaluate (4.16) (cf. [29]) as

$$
\text { const. } \begin{aligned}
& \frac{\Gamma(1 / 2(\alpha+\beta+1+i \lambda)) \Gamma(1 / 2(\alpha-\beta+1+i \lambda)) \Gamma(1 / 2(\delta+i \mu+1+i \lambda)) \Gamma(1 / 2(\delta-i \mu+1+i \lambda))}{\Gamma(i \lambda)} \\
& \times W_{n}\left(1 / 4 \lambda^{2} ; 1 / 2(\delta+i \mu+1), 1 / 2(\delta-i \mu+1), 1 / 2(\alpha+\beta+1), 1 / 2(\alpha-\beta+1)\right),
\end{aligned}
$$

where

$$
\begin{aligned}
& W_{n}\left(x^{2} ; a, b, c, d\right):=(a+b)_{n}(a+c)_{n}(a+d)_{n} \\
& \times_{4} F_{3}\left(\begin{array}{c}
-n, n+a+b+c+d-1, a+i x, a-i x \\
a+b, a+c, a+d
\end{array}\right)
\end{aligned}
$$

is a Wilson polynomial, a polynomial of degree $n$ in $x^{2}$ (cf. Wilson [37] except for the standardized notation which is taken from Askey and Wilson [4, Appendix]). If the parameters $a, b, c, d$ all have positive real parts and if they are all real or one or both pairs of them consist of complex conjugates then the functions $x \mapsto W_{n}\left(x^{2}\right)$ form a complete orthogonal system of functions on $\mathbb{R}_{+}$with respect to the weight function

$$
x \mapsto\left|\frac{\Gamma(a+i x) \Gamma(b+i x) \Gamma(c+i x) \Gamma(d+i x)}{\Gamma(2 i x)}\right|^{2} .
$$

So we have the identity

$$
(4.16)=(4.17) \text {, }
$$

where the constant in (4.17) is independent of $\lambda$, but does depend on $n, \alpha, \beta, \delta, \mu$. Observe that the orthogonality of the Jacobi polynomials and the isometry property of the Jacobi function transform imply the orthogonality relations for the Wilson polynomials. On the other hand the isometry property of the Jacobi function transform is implied by the orthogonality properties for the Jacobi and Wilson polynomials.

If $|\beta|>\alpha+1$ then one of the parameters of $W_{n}$ in (4.17) becomes negative. Then the discrete terms in the inversion formula for the Jacobi function transform will correspond to discrete components of the orthogonality measure for the Wilson polynomials. These discrete mass points do indeed occur if one of the four parameters in (4.18) is negative, cf. Wilson [37, (3.3)].

Just as (4.5) was a limit case of (4.4), we can take limits in the identity (4.20) by replacing $t$ by $\delta^{-1 / 2} t$ and $\lambda$ by $\delta^{1 / 2} \lambda$ and by letting $\delta \rightarrow \infty$. Then both the Jacobi polynomials and the Wilson polynomials tend to Laguerre polynomials, which are defined by

$$
L_{n}^{\alpha}(x):=\frac{(\alpha+1)_{n}}{n !}{ }_{1} F_{1}(-n, \alpha+1 ; x)
$$

and which are orthogonal polynomials on $\mathbb{R}_{+}$with respect to the weight function $x \mapsto x^{\alpha} e^{-x}$. Furthermore, Jacobi functions tend to Bessel functions 


$$
J_{\alpha}(x):=\frac{(1 / 2 x)^{\alpha}}{\Gamma(\alpha+1)}{ }_{0} F_{1}\left(\alpha+1 ;-1 / 4 x^{2}\right)
$$

and the Jacobi function transform pair to the Hankel transform pair

$$
\left.\begin{array}{l}
g(\lambda)=\int_{0}^{\infty} f(t) J_{\alpha}(\lambda t)(\lambda t)^{1 / 2} d t \\
f(t)=\int_{0}^{\infty} g(\lambda) J_{\alpha}(\lambda t)(\lambda t)^{1 / 2} d \lambda
\end{array}\right\}
$$

The limit identity (cf. [29]) is

$$
\int_{0}^{\infty} t^{\alpha+1 / 2} e^{-1 / 2 t^{2}} L_{n}^{\alpha}\left(t^{2}\right) J_{\alpha}(\lambda t)(\lambda t)^{1 / 2} d t=(-1)^{n} \lambda^{\alpha+1 / 2} e^{-1 / 2 \lambda^{2}} L_{n}^{\alpha}\left(\lambda^{2}\right) .
$$

This identity is quite classical, cf. [15, Vol.2, 8.9(3)].

We can summarize our results in Table 2 below.

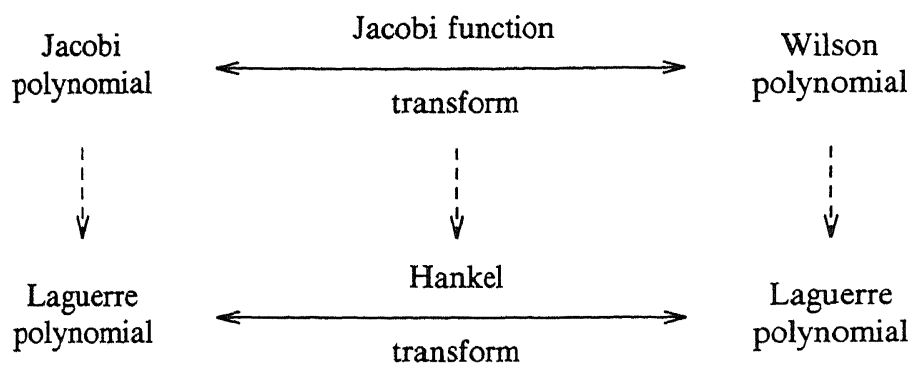

Table 2

Remark. There is another limit case of (4.20), in which Jacobi polynomials, Jacobi functions and Wilson polynomials tend to Laguerre polynomials, Whittaker functions of the second kind and continuous dual Hahn polynomials, respectively (cf. $[29, \S 5])$.

\section{The Askey scheme of hypergeometric orthogonal polynomials}

There are many indications that the Wilson polynomials, introduced in (4.18), are the most general orthogonal polynomials which can be written in terms of a $F_{q}$-hypergeometric function. In Table 3 below we reproduce Askey's scheme of hypergeometric orthogonal polynomials, as (almost) given in Askey and Wilson [4, Appendix]. The arrows denote limit transitions. Of course, shortcuts can be made in these limit transitions by at once going down more than one level.

Let us give the explicit expressions and weight functions of the orthogonal polynomials in the Askey scheme.

(a) Wilson polynomials. See (4.18), (4.19).

(b) Racah polynomials.

$$
\begin{array}{r}
R_{n}(\lambda(x) ; \alpha, \beta, \gamma, \delta):={ }_{4} F_{3}\left(\begin{array}{c}
-n, n+\alpha+\beta+1,-x, x+\gamma+\delta+1 \\
\alpha+1, \beta+\delta+1, \gamma+1
\end{array} \mid 1\right), \\
\lambda(x):=x(x+\gamma+\delta+1), \beta+\delta+1=-N, n=0,1, \ldots, N .
\end{array}
$$

The functions $x \mapsto R_{n}(\lambda(x))$ are orthogonal on $\{0,1, \ldots, N\}$ with respect to weights which can be explicitly given.

(c) Continuous dual Hahn polynomials.

$$
S_{n}\left(x^{2} ; a, b, c\right):=(a+b)_{n}(a+c)_{n} F_{2}\left(\begin{array}{c}
-n, a+i x, a-i x \\
a+b, a+c
\end{array} \mid 1\right),
$$


(a) Wilson

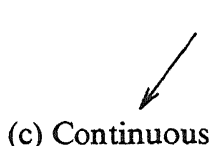
dual Hahn

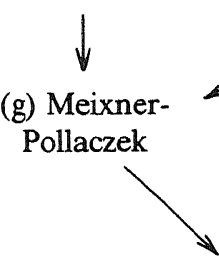

(k)

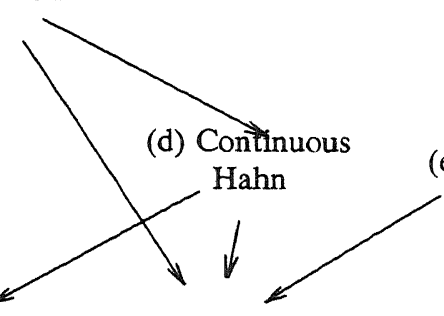

(h) Jacobi (b) Racah

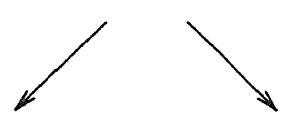

(e) Hahn

(f) dual Hahn

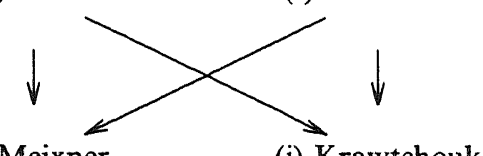

(i) Meixner

(j) Krawtchouk

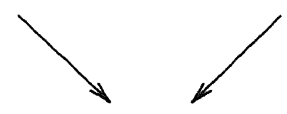

(1) Charlier

(m) Hermite

Table 3

where $a, b, c$ have positive real parts; if one of these parameters is not real then one of the other parameters is its complex conjugate. The functions $x \mapsto S_{n}\left(x^{2}\right)$ are orthogonal on $(0, \infty)$ with respect to the weight function

$$
x \mapsto\left|\frac{\Gamma(a+i x) \Gamma(b+i x) \Gamma(c+i x)}{\Gamma(2 i x)}\right|^{2} .
$$

(d) Continuous Hahn polynomials.

$$
p_{n}(x ; a, b, \bar{a}, \bar{b}):=i^{n} \frac{(a+\bar{a})_{n}(a+\bar{b})_{n}}{n !}{ }_{3} F_{2}\left(\begin{array}{c}
-n, n+a+\bar{a}+b+\bar{b}-1, a+i x \\
a+\bar{a}, a+\bar{b}
\end{array} \mid 1\right),
$$

where $a, b$ have positive real part. The polynomials $p_{n}$ are orthogonal on $\mathbb{R}$ with respect to the weight function $x \mapsto\rfloor\left.\Gamma(a+i x) \Gamma(b+i x)\right|^{2}$. In Askey and Wilson [4, Appendix] only the symmetric case $(a, b>0$ or $a=b)$ of these polynomials occurs. Under these restrictions on $a, b$, the polynomials (5.4) reduce to (2.14), up to a constant factor. The general continuous Hahn polynomials were discovered by Atakishiyev and Suslov [5]. See Askey [2] for notation, but read $a+i x$ instead of $a-i x$ in his formula (3).

(e) Hahn polynomials.

$$
Q_{n}(x ; \alpha, \beta, N):={ }_{3} F_{2}\left(\begin{array}{c|c}
-n, n+\alpha+\beta+1,-x \\
\alpha+1,-N
\end{array}\right), \quad n=0,1, \ldots, N,
$$

orthogonal on $\{0,1, \ldots, N\}$ with respect to the weights

$$
x \mapsto\left(\begin{array}{c}
\alpha+x \\
x
\end{array}\right]\left[\begin{array}{c}
N-x+\beta \\
N-x
\end{array}\right) .
$$

(f) Dual Hahn polynomials.

$$
R_{n}(x(x+\alpha+\beta+1) ; \alpha, \beta, N):={ }_{3} F_{2}\left(\begin{array}{c}
-x, x+\alpha+\beta+1,-n \mid 1 \\
\alpha+1,-N
\end{array}\right), \quad n=0,1, \ldots, N,
$$

i.e., as (5.5) but with $n$ and $x$ interchanged. The functions $x \mapsto R_{n}(x(x+\alpha+\beta+1))$ are orthogonal on $\{0,1, \ldots, N\}$ with respect to weights which can be explicitly given.

(g) Meixner-Pollaczek polynomials. 


$$
P_{n}^{(a)}(x ; \phi):=e_{2}^{i n \phi} F_{1}\left(-n, a+i x ; 2 a ; 1-e^{-2 i \phi}\right), \quad a>0,0<\phi<\pi,
$$

orthogonal on $\mathbb{R}$ with respect to the weight function $x \mapsto e^{(2 \phi-\pi) x}|\Gamma(a+i x)|^{2}$.

(h) Jacobi polynomials. See (4.6).

(i) Meixner polynomials.

$$
M_{n}(x ; \beta, c):={ }_{2} F_{1}\left(-n,-x ; \beta ; 1-c^{-1}\right), \quad 0<c<1, \beta>0,
$$

orthogonal on $\mathbb{Z}_{+}$with respect to the weights $x \mapsto(\beta)_{x} c^{x} / x$ !.

(j) Krawtchouk polynomials.

$$
K_{n}(x ; p, N):={ }_{2} F_{1}\left(-n,-x ;-N ; p^{-1}\right), \quad 0<p<1, n=0,1, \ldots, N,
$$

orthogonal on $\{0,1, \ldots, N\}$ with respect to the weights

$$
x \mapsto\left[\begin{array}{c}
N \\
x
\end{array}\right] p^{x}(1-p)^{N-x} .
$$

(k) Laguerre polynomials. See (4.21).

(l) Charlier polynomials.

$$
C_{n}(x ; a):={ }_{2} F_{0}\left(-n,-x ;-a^{-1}\right), \quad a>0,
$$

orthogonal on $\mathbb{Z}_{+}$with respect to the weights $x \mapsto a^{x} / x !$.

(m) Hermite polynomials.

$$
H_{n}(x):=(2 x)^{n}{ }_{2} F_{0}\left(-1 / 2 n, 1 / 2(1-n) ;-x^{-2}\right),
$$

orthogonal on $\mathbb{R}$ with respect to the weight function $x \mapsto e^{-x^{2}}$.

Note that the number of parameters on which the families of orthogonal polynomials depend, declines from 4 to 0 as one goes down in Table 3. The families in the left half of the table (including the Hermite polynomials) have continuous weight functions, while the ones in the right half have discrete orthogonality measure. Jacobi, Laguerre and Hermite polynomials are the hard-core classical orthogonal polynomials, but most of the other families also have a long history. Only Wilson, Racah, continuous Hahn and continuous dual Hahn polynomials are recent inventions. See Andrews and Askey [1] for more information about the concept "classical orthogonal polynomial".

The limit behaviour as shown in Table 3 is oversimplified, since, in fact, there are three types of Wilson polynomials $W_{n}\left(x^{2} ; a, b, c, d\right)$ (cf. (4.18)), namely the type $(a, b, c, d)$, where $a, b, c, d$ are real, the type $(a, \bar{a}, c, d)$, where $c, d$ are real, and the type $(a, \bar{a}, c, \bar{c})$, where we have two pairs of complex conjugates. (Of course there are overlaps.) There are also two types of continuous dual Hahn polynomials $S_{n}\left(x^{2} ; a, b, c\right)$ (cf. (5.2)): the type $(a, b, c)$ with $a, b, c$ real and the type $(a, \bar{a}, c)$. With this refinement the left half of Table 3 can be rewritten as Table 4 below.

Each system of orthogonal polynomials $p_{n}(x)$ in the Askey scheme can be considered as an orthogonal basis $\left\{p_{n}\right\}$ of some Hilbert space. After trivial transformations these Hilbert spaces take the form $L^{2}(\mathbb{R}), L^{2}\left(\mathbb{Z}_{+}\right)$or $L^{2}(\{1, \ldots, N\})$. Expansion of an arbitrary element of the Hilbert space in terms of the basis can be considered as an isometry of $L^{2}(\mathbb{R})$ or $L^{2}\left(\mathbb{Z}_{+}\right)$onto $L^{2}\left(\mathbb{Z}_{+}\right)$or of $L^{2}(\{1, \ldots, N\})$ onto itself. The limit transitions in Tables 3 and 4 can now be considered as taking weak limits of the isometries. Sometimes the two Hilbert spaces do not change in this limit transition, but sometimes they do, cf. in Table 3:

$(\mathrm{e}) \rightarrow(\mathrm{i}),(\mathrm{f}) \rightarrow(\mathrm{i}),(\mathrm{j}) \rightarrow(\mathrm{l})$ and $(\mathrm{e}) \rightarrow(\mathrm{h})\left(\right.$ from $L^{2}(\{1, \ldots, N\})$ to $L^{2}\left(\mathbb{Z}_{+}\right)$once or twice);

(e) $\rightarrow$ (h) (from $L^{2}(\{1, \ldots, N\})$ to $\left.L^{2}(\mathbb{R})\right)$;

$(\mathrm{l}) \rightarrow(\mathrm{m})$ and $(\mathrm{i}) \rightarrow(\mathrm{k})\left(\right.$ from $L^{2}\left(\mathbb{Z}_{+}\right)$to $L^{2}(\mathbb{R})$ ).

Schematically the possibilities for types of isometries and limit transitions are given in Table 5 below.

This table strongly suggests that isometries of $L^{2}(\mathbb{R})$ onto itself might also be obtained as limit cases of isometries occurring in the Askey scheme. The following example shows that this is indeed 


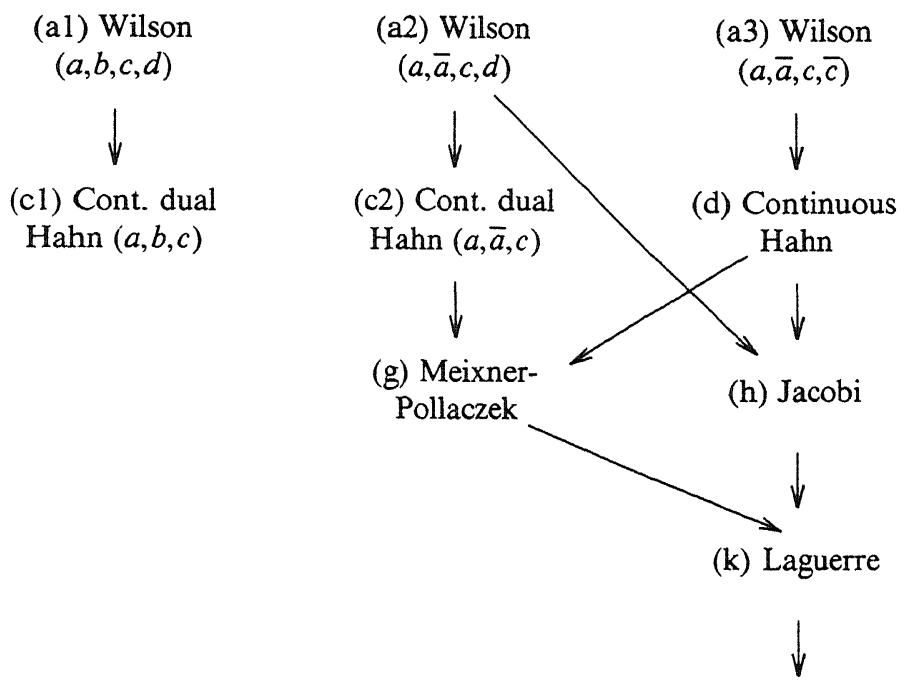

(m) Hermite

Table 4

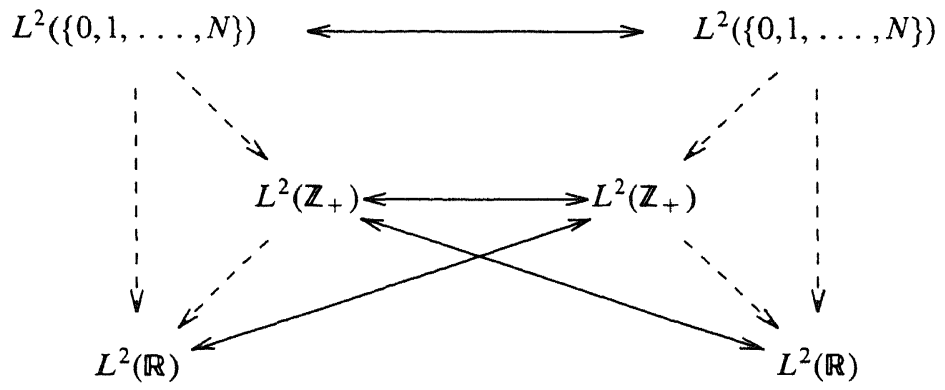

Table 5

possible, at least formally. We have

$$
\begin{aligned}
& \lim _{n \rightarrow \infty}{ }_{3} F_{2}\left(\begin{array}{c}
-n, 1 / 2(\alpha+\beta+1)+i \lambda, 1 / 2(\alpha+\beta+1)-i \lambda \\
1 / 2(\alpha+\beta+1)+n \operatorname{sh}^{-2} t, \alpha+1
\end{array} \mid 1\right) \\
& ={ }_{2} F_{1}\left(1 / 2(\alpha+\beta+1)+i \lambda, 1 / 2(\alpha+\beta+1)-i \lambda ; \alpha+1 ;-\operatorname{sh}^{2} t\right),
\end{aligned}
$$

where the limit is taken formally by power series expansion and termwise limits. Hence, in view of (5.2) and (4.7) we obtain

$$
\lim _{n \rightarrow \infty} \frac{S_{n}\left(1 / 4 \lambda^{2} ; 1 / 2(\alpha+\beta+1), n \operatorname{sh}^{-2} t, 1 / 2(\alpha-\beta+1)\right)}{\left(1 / 2(\alpha+\beta+1)+n \operatorname{sh}^{-2} t\right)_{n}(\alpha+1)_{n}}=\phi_{\lambda}^{(\alpha, \beta)}(t)
$$

i.e., Jacobi functions are limits of continuous dual Hahn polynomials. In $\$ 7$ we will find many more examples of unitary integral transforms with hypergeometric kernel as limits of hypergeometric orthogonal polynomial transforms and we will be able to give an extension of the Askey scheme such that these integral transforms are also incorporated. Moreover, in $\S 6$ we will find isometries of $L^{2}(\mathbb{Z})$ and $L^{2}\left(\mathbb{Z}_{+}\right)$which are of hypergeometric but not of orthogonal polynomial type. 


\section{Orthogonal special functions from group theory: two fundamental constructions}

Let $G$ be a Lie group and $\pi$ a unitary representation of $G$ on a Hilbert space $\mathcal{H}(\pi)$ (cf. §3). Let $H$ and $L$ be closed subgroups of $G$ and suppose that the restrictions of $\pi$ to $H$ and $L$ are multiplicity free. Then we can write $\pi$ restricted to $H$ as a direct sum or integral of irreducible representations $\gamma$ of $H$ uniquely, and similarly for $\delta$, cf. Table 6 below.

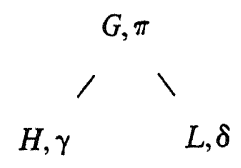

Table 6

In the very special situation that all these representations $\gamma$ of $H$ and $\delta$ of $L$ are one-dimensional, we have two orthonormal bases for $\mathcal{H}(\pi)$ (in the ordinary sense in case of a direct sum and in the generalized sense, with distribution vectors, in case of a direct integral). We write these bases as $\left\{v_{\pi, \gamma}\right\}$ with $\gamma$ in some subset of $\hat{H}$ and $\left\{w_{\pi, \delta}\right\}$ with $\delta$ in some subset of $\hat{L}$, such that

$$
\pi(h) v_{\pi, \gamma}=\gamma(h) v_{\pi, \gamma}, \quad \pi(l) w_{\pi, \delta}=\delta(l) w_{\pi, \delta}
$$

There are now two ways to construct orthogonal special functions adapted to the group $G$ and its subgroups $H$ and $L$.

Method I.

$$
\phi^{\gamma, \delta}(g, \pi):=\left(\pi(g) v_{\pi, \gamma}, w_{\pi, \delta}\right), \quad g \in G, \pi \in \hat{G}, \gamma \in \hat{H}, \delta \in \hat{L}
$$

Here $\gamma, \delta$ are considered as parameters which are held fixed, while we get orthogonality and dual orthogonality with respect to the variables $g$ and $\pi$, respectively. In case of a compact group $G$, orthogonality of the functions $\phi^{\gamma, \delta}(., \pi)$ is just the Schur orthogonality for matrix elements of irreducible representations and completeness of the system follows from the Peter-Weyl theorem. In case of a noncompact group $G$, the integral transform with $\phi^{\gamma, \delta}(g, \pi)$ as kernel is a specialization of the Fourier transform on the group and the inversion formula is a particular case of the Plancherel formula on the group. Part of the game in all these cases is the restriction of $\phi^{\gamma, \delta}(., \pi)$ to a suitable subset of $G$ which (for the particular $\gamma, \delta$ ) already completely determines the function. If this subset can be identified with a subset of Euclidean space and the essential part of the Haar measure for $G$ can be determined on this subset, then we have made the identification of the orthogonal system (6.2) with a system which can be described without group theoretic terminology.

If $v_{\pi, \gamma}$ and $w_{\pi, \delta}$ are generalized elements of $\mathcal{H}(\pi)$ then the inner product in (6.2) can be given a meaning as a distribution by testing it against $C^{\infty}$-functions $f$ with compact support on $G$. This testing yields $\left(\pi(f) v_{\pi, \gamma}, w_{\pi, \delta}\right)$, then $\pi(f) v_{\pi, \gamma}$ is a $C^{\infty}$-vector and its inner product with the distribution vector $w_{\pi, \delta}$ is well-defined.

\section{Method II}

$$
\psi^{\xi, \pi}(\gamma, \delta):=\left(\pi(g) v_{\pi, \gamma}, w_{\pi, \delta}\right), \quad g \in G, \pi \in \hat{G}, \gamma \in \hat{H}, \delta \in \hat{L} .
$$

Now $g$ and $\pi$ are held fixed as parameters, while $\gamma$ and $\delta$ are the variables with respect to which orthogonality and dual orthogonality are obtained. Note that $\left\{\pi(g) v_{\pi, \gamma}\right\}$ and $\left\{w_{\pi, \delta}\right\}$ are both (generalized) orthonormal bases of $\mathcal{S}(\pi)$, so $\psi^{\xi, \pi}(\gamma, \delta)$ is the matrix element or integral kernel of the unitary transformation which maps the one basis onto the other.

Our requirement that the representations $\gamma$ and $\delta$ are one-dimensional is too restrictive, although it is surprising how many special functions already come out with this assumption, mainly for $G=S L(2, \mathbb{R}), S U(2)$ or $I_{0}\left(\mathbb{R}^{2}\right)$. More generally we assume that we have two chains of closed subgroups and corresponding irreducible representations, cf. Table 7 below.

Here we assume that the representation $\gamma_{i}$ of $H_{i}$, when restricted to $H_{i+1}$, has a multiplicity free decomposition in terms of irreducible representations $\gamma_{i+1}$ of $H_{i+1}$, and the irreducible 


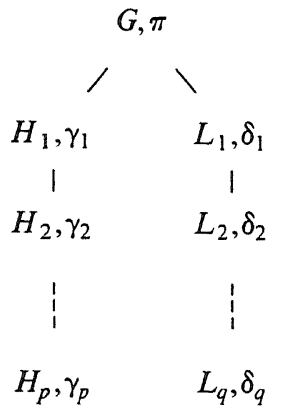

Table 7

representations $\gamma_{p}$ of $H_{p}$ which occur are one-dimensional, and similarly for the other chain. Thus we obtain (generalized) orthonormal bases $\left\{v_{\pi, \gamma_{1}, \ldots, \gamma_{p}}\right\}$ and $\left\{w_{\pi, \delta_{1}}, \ldots, \delta_{q}\right\}$ for $\mathcal{H}(\pi)$ and we can write, on the one hand,

$$
\phi^{\gamma_{1}}, \ldots, \gamma_{p} ; \delta_{1}, \ldots, \delta_{q}(g, \pi):=\left(\pi(g) v_{\pi, \gamma_{1}}, \ldots, \gamma_{p}, w_{\pi, \delta_{1}}, \ldots, \delta_{q}\right),
$$

where $\gamma_{1}, \ldots, \gamma_{p}, \delta_{1}, \ldots, \delta_{q}$ are considered as parameters, and, on the other hand,

$$
\psi^{g, \pi}\left(\gamma_{1}, \ldots, \gamma_{p} ; \delta_{1}, \ldots, \delta_{q}\right):=\left(\pi(g) v_{\pi, \gamma_{1}}, \ldots, \gamma_{p}, w_{\pi, \delta_{1}}, \ldots, \delta_{q}\right),
$$

where $g, \pi$ are considered as parameters.

Let us discuss a number of special cases of the constructions I and II.

Ia. Let $K:=H=L$ be compact and let $(G, K)$ be a Gelfand pair, i.e., in each $\pi \in \hat{G}$ the trivial representation 1 of $K$ occurs at most once. Take $\gamma=\delta=1$ in $(6.2)$. Then $\phi^{1,1}(., \pi)$ becomes a spherical function for $(G, K)$, cf. (3.1).

Ib. Let $K:=H_{1}=L_{1}$ be compact and take $p=1, \gamma_{1}=1$ in (6.4). Then $\phi^{1 ; \delta_{1}, \ldots, \delta_{q}}$ becomes an associated spherical function for $(G, K)$, cf. (3.2).

Ic. Let $H$ and $L$ be non-conjugate subgroups of $G$, let $H$ be compact and take $\gamma=1=\delta$ in (6.2). Then $\phi^{1,1}(., \pi)$ is a so-called intertwining function for the triple $(G, H, L)$.

Id. Let $H_{i}=L_{i}(i=1, \ldots, p ; p=q)$. Then $\phi^{\gamma_{1}, \ldots, \gamma_{p} ; \delta_{1}, \ldots, \delta_{p}}(., \pi)$ as given by (6.4) denotes a (generalized) matrix element of $\pi$ with respect to the basis $\left\{v_{\pi, \gamma_{1}, \ldots, \gamma_{p}}\right\}$, with the dependence on $g$ emphasized.

IIa. Assumptions as in case Id. The function $\psi^{g, \pi}$ in (6.5) is again a (generalized) matrix element of $\pi$, but here with the dependence on $\gamma_{1}, \ldots, \gamma_{p}$ and $\delta_{1}, \ldots, \delta_{p}$ emphasized.

IIb. Let $H$ and $L$ be non-conjugate subgroups. Then $\psi^{g, \pi}$ as given by $(6.5)$ is called a mixed basis matrix element (possibly generalized) of $\pi$. Note that, without loss of generality, we may assume $g=e$ in (6.5). Then the functions $\psi^{e, \pi}$ in (6.5) are called the overlap functions for the bases $\left\{v_{\pi, \gamma_{1}}, \ldots, \gamma_{p}\right\}$ and $\left\{w_{\pi, \delta_{1}}, \ldots, \delta_{q}\right\}$ of $\mathcal{H}(\pi)$.

The spherical function case Ia is already so rich that whole books can be written only about this case. See for instance Helgason [21], Faraut [18] and Koornwinder [27]. Let us here just make the observation that spherical functions always satisfy a product formula

$$
\phi\left(g_{1}, \pi\right) \phi\left(g_{2}, \pi\right)=\int_{K} \phi\left(g_{1} k g_{2}\right) d k
$$

with corresponding commutative positive convolution structure for the $K$-biinvariant functions on $G$ and a linearization formula

$$
\phi\left(g, \pi_{1}\right) \phi\left(g, \pi_{2}\right)=\int_{\hat{G}} \phi\left(g, \pi_{3}\right) c\left(\pi_{1}, \pi_{2}, \pi_{3}\right) d \mu\left(\pi_{3}\right)
$$


with $c\left(\pi_{1}, \pi_{2}, \pi_{3}\right) \geqslant 0$ and corresponding positive dual convolution structure. If we can identify the spherical functions with special functions then we have, of course, also two positive convolution structures associated with the expansions in terms of these special functions.

In case II we may have the situation of a third subgroup chain. In the simple situation we started with in this section, for instance, we may have a diagram as in Table 8 below and three corresponding (generalized) orthonormal bases $\left\{u_{\pi, \beta}\right\},\left\{v_{\pi, \gamma}\right\}$ and $\left\{w_{\pi, \delta}\right\}$.

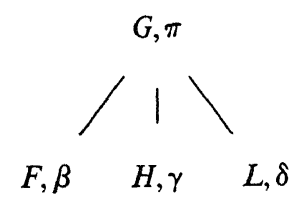

Table 8

Then the three functions of type (6.3) (with $g=e$ ) related to these bases satisfy an identity of the form

$$
\left(u_{\pi, \beta}, w_{\pi, \delta}\right)=\int_{\hat{H}}\left(u_{\pi, \beta}, v_{\pi, \gamma}\right)\left(v_{\pi, \gamma}, w_{\pi, \delta}\right) d \mu(\gamma) .
$$

In section 7 we will meet concrete examples of this.

Mixed case. There is a third way to obtain orthogonal systems from the inner products $\left(\pi(g) v_{\pi, \gamma}, w_{\pi, \delta}\right)$. Fix $\pi$ and $\gamma$ as parameters and consider

$$
\chi^{\pi, \gamma}(g, \delta):=\left(\pi(g) v_{\pi, \gamma}, w_{\pi, \delta}\right)
$$

We might as well fix $\pi$ and some $u \in \mathcal{G}(\pi)$ and then consider

$$
\chi_{1}^{\pi, u}(g, \gamma):=\left(\pi(g) u, v_{\pi, \gamma}\right), \quad \chi_{2}^{\pi, u}(g, \delta):=\left(\pi(g) u, w_{\pi, \delta}\right) .
$$

Then a Schur type orthogonality yields

$$
\int_{G} \chi_{1}^{\pi, u}(g, \gamma) \overline{\chi_{2}^{\pi, u}(g, \delta)} d g=\text { const. }\left(w_{\pi, \delta}, v_{\pi, \gamma}\right) \text {. }
$$

Again we have produced an identity involving three orthogonal systems: two of mixed type and one of type II. It will turn out later that (4.16), (4.17) can be brought into this form.

As an example of (6.2) versus (6.3) consider $G:=S U(2)$, the group of $2 \times 2$ unitary matrices with determinant 1. (See Vilenkin $[36, C h .3]$ as a reference for this example.) Let $K:=H=L=U(1)$, the subgroup of diagonal matrices in $S U(2)$. For each $l \in 1 / 2 \mathbb{Z}_{+}$there is, up to equivalence, a unique irreducible unitary representation $T^{l}$ of $G$ with $(2 l+1)$-dimensional representation space $\mathscr{T}\left(T^{l}\right)$. We can choose an orthonormal $K$-basis $\left\{v_{-l}, v_{-l+1}, \ldots, v_{l}\right\}$ of $\mathcal{H C}\left(T^{l}\right)$ such that

$$
T^{l}\left(\operatorname{diag}\left(e^{-1 / 2 i \theta}, e^{1 / 2 i \theta}\right)\right) v_{n}=e^{i n \theta} v_{n}, \quad n=-l,-l+1, \ldots, l .
$$

Then $\left(T^{l}(g) v_{n}, v_{m}\right)$ as a function of $g \in G$ is already completely determined if we know it for $g$ restricted to the matrices

$$
u_{\gamma}:=\left[\begin{array}{rr}
\cos 1 / 2 \gamma & -\sin 1 / 2 \gamma \\
\sin 1 / 2 \gamma & \cos 1 / 2 \gamma
\end{array}\right] .
$$

So write

$$
\phi^{n, m}\left(u_{\gamma}, T^{l}\right):=\left(T^{l}\left(u_{\gamma}\right) v_{n}, v_{m}\right)=: \psi^{\gamma, l}(n, m) .
$$

Then the functions $\phi^{n, m}$ (fitting into case Id) can be evaluated in terms of Jacobi polynomials (cf. (4.6)) by

$$
\phi^{n, m}\left(u_{\gamma}, T^{l}\right)=\left[\frac{(l-m) !(l+m) !}{(l-n) !(l+n) !}\right]^{1 / 2}
$$




$$
\times(\sin 1 / 2 \gamma)^{m-n}(\cos 1 / 2 \gamma)^{m+n} P_{l-m}^{(m-n, m+n)}(\cos \gamma), \quad m+n, m-n \geqslant 0 .
$$

Orthogonality of the Jacobi polynomials is here an immediate consequence of Schur's orthogonality relations for matrix elements of the inequivalent irreducible representations $T^{l}$.

On the other hand, as was shown in [26, §2], the functions $\psi^{\gamma, l}$ (fitting into case IIa) can be evaluated in terms of Krawtchouk polynomials (cf. (5.10)) by

$$
\begin{aligned}
& \psi^{\gamma, l}(n, m)=\left[\begin{array}{c}
2 l \\
l+m
\end{array}\right)^{1 / 2}\left(\begin{array}{c}
2 l \\
l+n
\end{array}\right)^{1 / 2} \\
& \times(-1)^{l+n}(\sin 1 / 2 \psi)^{n+m+2 l}(\cos 1 / 2 \psi)^{-n-m} K_{m+l}\left(n+l ; \sin ^{2} 1 / 2 \psi, 2 l\right) .
\end{aligned}
$$

The orthogonality of the Krawtchouk polynomials is now an immediate consequence of the fact that $T^{l}\left(u_{\gamma}\right)$ is a unitary operator and the basis $\left\{v_{n}\right\}$ is orthonormal.

It is possible to deform the group $S U(2)$ such that it tends to the Euclidean motion group $I_{0}\left(\mathbb{R}^{2}\right)$, while the subgroup $U(1)$ of $S U(2)$ becomes the subgroup $S O(2)$ of $I_{0}\left(\mathbb{R}^{2}\right)$ and the matrices $u_{\gamma}$ tend to the translations $t_{x}$. Then the irreducible representations $T^{l}$ of $S U(2)$ also tend to an irreducible unitary representation $\pi_{\lambda}$ of $I_{0}\left(\mathbb{R}^{2}\right)$ (cf. the representation $\pi$ of $I_{0}\left(\mathbb{R}^{2}\right)$ which we met in section 3). The orthonormal basis $\left\{v_{-l}, v_{-l+1}, \ldots, v_{l}\right\}$ of $\mathcal{S}\left(T^{l}\right)$ tends to an orthonormal basis $\left\{v_{n}\right\}_{n \in \mathbf{Z}}$ of $\mathcal{H}\left(\pi_{\lambda}\right)$ which behaves nicely with respect to the subgroup $S O(2)$ of $I_{0}\left(\mathbb{R}^{2}\right)$. We obtain, by taking limits in (6.14) or (6.15) that

$$
\lim _{l \rightarrow \infty}\left(T^{l \lambda}\left(u_{x} / l\right) v_{n}, v_{m}\right)=\left(\pi_{\lambda}\left(t_{x}\right) v_{n}, v_{m}\right)=(-1)^{m-n} J_{n+m}(\lambda x)
$$

where $J_{n+m}$ is a Bessel function (cf. (2.1)). When we emphasize the dependence on $\lambda, x$ in $J_{n+m}(\lambda x)$ then we get the generalized orthogonal system which yields the integral kernel for the Hankel transform (cf. (4.23)). However, when we emphasize the dependence on $n$ and $m$ then we get the extremely simple orthogonality relations

$$
\sum_{n=-\infty}^{\infty} J_{n+k}(x) J_{n+l}(x)=\delta_{k, l}, \quad k, l \in \mathbb{Z}, x \in \mathbb{R},
$$

which are not widely known, although they are hidden in the formula books. Indeed, start with

$$
e^{i x \sin \phi}=\sum_{n=-\infty}^{\infty} e^{i n \phi} J_{n}(x)
$$

(the Fourier series expansion implied by (2.2)). Hence

$$
e^{i x \sin \phi} e^{-i k \phi}=\sum_{n=-\infty}^{\infty} e^{i n \phi} J_{n+k}(x) \text {. }
$$

So, by Parseval's formula for Fourier series,

$$
\sum_{n=-\infty}^{\infty} J_{n+k}(x) J_{n+l}(x)=(2 \pi)^{-1} \int_{0}^{2 \pi} e^{i x \sin \phi} e^{-i k \phi} \overline{e^{i x \sin \phi} e^{-i l \phi}} d \phi=\delta_{k, l}
$$

By (6.17) we have, for each $x \in \mathbb{R}$, an orthonormal system $\left\{n \mapsto J_{n+k}(x)\right\}_{k \in \mathbf{Z}}$ in $L^{2}(\mathbb{Z})$, which consists of ${ }_{0} F_{1}$-hypergeometric functions and is a limit case of the Krawtchouk polynomials, but is definitely not a system of orthogonal polynomials. Still it naturally fits into an extended concept of the Askey scheme.

One may wonder what else might be included in such an extended scheme. Here is another example, not (yet) coming from group theory. From the Jacobi polynomials we build two orthogonal bases of $L^{2}((0,1))$. A simple computation yields the transition matrix:

$$
\begin{aligned}
& \int_{0}^{1} x^{1 / 2 \alpha}(1-x)^{1 / 2 \beta} P_{n}^{(\alpha, \beta)}(1-2 x) x^{1 / 2 \alpha}(1-x)^{1 / 2 \delta} P_{m}^{(\alpha, \delta)}(1-2 x) d x \\
& =\frac{(-1)^{n}(\beta+1)_{n} \Gamma(\alpha+m+1) \Gamma(1 / 2(\beta+\delta)+1)(-m+1 / 2(\beta-\delta)+1)_{m}}{n ! m ! \Gamma(\alpha+1 / 2(\beta+\delta)+2+m)}
\end{aligned}
$$




$$
\times_{4} F_{3}\left(\begin{array}{c}
-n, n+\alpha+\beta+\delta+1,1 / 2(\beta+\delta)+1,1 / 2(\beta-\delta)+1 \\
\beta+1, m+\alpha+1 / 2(\beta+\delta)+2,-m+1 / 2(\beta-\delta)+1
\end{array} \mid 1\right)
$$

This yields an orthogonal system on $L^{2}\left(\mathbb{Z}_{+}\right)$of hypergeometric but non-polynomial type. One can derive a limit case of (2.8) with Laguerre polynomials at the left hand side and $a_{3} F_{2}$ of argument 1 at the right hand side.

\section{Orthogonal special functions related to the discrete series of $S L(2, \mathbb{R})$}

The paper [9] by Basu and Wolf is a rich source of special functions related to representations of $S L(2, \mathbb{R})$ (the group of real $2 \times 2$ matrices of determinant 1 ). In particular, the special functions related to the discrete series of $S L(2, \mathbb{R})$, discussed in [9, section 3], are very neat, much related to the Askey scheme and a good illustration of the framework we developed in $\S 6$. I feel justified to reproduce here some of the formulas in [9], since the orthogonality properties, limit relations and identifications with known special functions are not much emphasized there.

The discrete series representations $D_{k}^{+}(k=1 / 2,1,3 / 2, \cdots)$ of $S L(2, \mathbb{R})$ are realized in $[9,(2.5)]$ as unitary representations on $L^{2}\left(\mathbb{R}_{+}\right)$:

$$
\begin{aligned}
& \left(D_{k}^{+}\left[\begin{array}{ll}
a & b \\
c & d
\end{array}\right] f\right)(r):=\int_{0}^{\infty} f(s) e^{-i \pi k} b^{-1}(r s)^{1 / 2} \\
& \times \exp \left(1 / 2 i b^{-1}\left(d r^{2}+a s^{2}\right)\right) J_{2 k-1}\left(b^{-1} r s\right) d s, \quad f \in L^{2}\left(\mathbb{R}_{+}\right), b \neq 0,
\end{aligned}
$$

and, by taking the limit as $b \rightarrow 0$ :

$$
\left(D_{k}^{+}\left(\begin{array}{ll}
a & 0 \\
c & d
\end{array}\right) f\right)(r):=(\operatorname{sgn} a)^{2 k}|a|^{-1 / 2} e^{1 / 2 i a^{-1} c r^{2}} f\left(|a|^{-1} r\right), \quad f \in L^{2}\left(\mathbb{R}_{+}\right) .
$$

Further specializations of (7.1) and (7.2) yield:

$$
\begin{aligned}
& \left(D_{k}^{+}\left[\begin{array}{ll}
1 & 0 \\
c & 1
\end{array}\right] f\right)(r)=e^{1 / i i c r^{2}} f(r), \quad f \in L^{2}\left(\mathbb{R}_{+}\right), \\
& \left(D_{k}^{+}\left[\begin{array}{cc}
a & 0 \\
0 & a^{-1}
\end{array}\right] f\right)(r)=(\operatorname{sgn} a)^{2 k}|a|^{-1 / 2} f\left(|a|^{-1} r\right), \quad f \in L^{2}\left(\mathbb{R}_{+}\right), \\
& \left(D_{k}^{+}\left[\begin{array}{cc}
\cos ^{1 / 2} \gamma & -\sin 1 / 2 \gamma \\
\sin ^{1 / 2 \gamma} & \cos 1 / 2 \gamma
\end{array}\right] f\right)(r)=\int_{0}^{\infty} f(s) e^{i \pi k}\left(\sin ^{1 / 2 \gamma}\right)^{-1}(r s)^{1 / 2} \\
& \quad \times e^{-1 / 2 i(\operatorname{cotg} 1 / 2 \gamma)\left(r^{2}+s^{2}\right)} J_{2 k-1}\left((\sin 1 / 2 \gamma)^{-1} r s\right) d s, \quad f \in L^{2}\left(\mathbb{R}_{+}\right), \gamma \notin 2 \pi \mathbb{Z}, \\
& \left(D_{k}^{+}\left[\begin{array}{cc}
0 & -1 \\
1 & 0
\end{array}\right] f\right)(r)=\int_{0}^{\infty} f(s) e^{i \pi k} J_{2 k-1}(r s)(r s)^{1 / 2} d s, \quad f \in L^{2}\left(\mathbb{R}_{+}\right) .
\end{aligned}
$$

The transformation (7.1) is built up from the Hankel transform (7.6) (cf. (4.23)) and the elementary transforms (7.3) and (7.4) according to the Bruhat decomposition of $\operatorname{SL}(2, \mathbb{R})$ :

$$
\left[\begin{array}{ll}
a & b \\
c & d
\end{array}\right]=\left(\begin{array}{rc}
-b & 0 \\
0 & -b^{-1}
\end{array}\right]\left[\begin{array}{cc}
1 & 0 \\
b d & 1
\end{array}\right]\left[\begin{array}{rr}
0 & -1 \\
1 & 0
\end{array}\right]\left[\begin{array}{cc}
1 & 0 \\
b^{-1} a & 1
\end{array}\right], a d-b c=1, b \neq 0 .
$$

Write $G:=S L(2, \mathbb{R})$. There are three conjugacy classes of 1-parameter subgroups in $G$ : the parabolic, hyperbolic and elliptic ones. We choose representatives of these conjugacy classes as follows:

$$
\begin{aligned}
& \text { parabolic: } N:=\left\{n_{c}:=\left[\begin{array}{ll}
1 & 0 \\
c & 1
\end{array}\right] \mid c \in \mathbb{R}\right\} ; \\
& \text { hyperbolic: } H:=\left\{h_{\beta}:=\left[\begin{array}{cc}
e^{-1 / 2 \beta} & 0 \\
0 & e^{1 / 2 \beta}
\end{array}\right] \mid \beta \in \mathbb{R}\right\} ;
\end{aligned}
$$




$$
\text { elliptic : } K:=\left\{u_{\gamma}:=\left[\begin{array}{rr}
\cos 1 / 2 \gamma & -\sin 1 / 2 \gamma \\
\sin 1 / 2 \gamma & \cos 1 / 2 \gamma
\end{array}\right] \mid \gamma \in \mathbb{R}\right\} .
$$

Following [9] we choose for $L^{2}\left(\mathbb{R}_{+}\right)=\mathcal{H}\left(D_{k}^{+}\right)$a generalized orthonormal $N$-basis $\left\{v_{k, \rho}^{N} \mid \rho \in \mathbb{R}_{+}\right\}$and $H$-basis $\left\{v_{k, \mu}^{H} \mid \mu \in \mathbb{R}\right\}$ and an orthonormal $K$-basis $\left\{v_{k, m}^{K} \mid m=k, k+1, \cdots\right\}$ such that

$$
\begin{array}{ll}
D_{k}^{+}\left(n_{c}\right) v_{k, \rho}^{N}=e^{i c \rho^{2}} v_{k, \rho}^{N}, & \rho \in \mathbb{R}_{+}, n_{c} \in N, \\
D_{k}^{+}\left(h_{\beta}\right) v_{k, \mu}^{H}=e^{i \beta \mu} v_{k, \mu}^{H}, & \mu \in \mathbb{R}, h_{\beta} \in H, \\
D_{k}^{+}\left(u_{\gamma}\right) v_{k, m}^{K}=e^{i \gamma m} v_{k, m}^{K}, \quad m=k, k+1, \cdots, u_{\gamma} \in K .
\end{array}
$$

Thus we are in the situation of Table 8 and the equations (7.8),(7.9),(7.10t, are examples of (6.1). See Table 9 below.

$\begin{array}{cccc} & & & \\ \text { subgroups: } & N & & \\ \text { labels of representations: } & \rho & \mu & m \\ \begin{array}{c}\text { corresponding } \\ \text { basis vectors: }\end{array} & v_{k, \rho}^{N} & v_{k, \mu}^{H} & v_{k, m}^{K}\end{array}$

Table 9

For the $N$-basis we have

$$
v_{k, \rho}^{N}:=\delta_{\rho}, \quad \rho \in \mathbb{R}_{+},
$$

where $\delta_{\rho}$ denotes the delta distribution with support $\{\rho\}$. Then (7.8) follows from (7.3).

For the $H$-basis we have

$$
v_{k, \mu}^{H}(r):=\pi^{-1 / 2} r^{-1 / 2+2 i \mu}, \quad r>0, \mu \in \mathbb{R} .
$$

Then (7.9) follows from (7.2).

For the $K$-basis we have an expression in terms of Laguerre polynomials:

$$
v_{k, m}^{K}(r):=\left[\frac{2(m-k) !}{(m+k+1) !}\right]^{1 / 2} r^{2 k-1 / 2} e^{-1 / 2 r^{2}} L_{m-k}^{2 k-1}\left(r^{2}\right), \quad m=k, k+1, \cdots .
$$

Then (7.10) can be obtained from the following generalization of (4.24) (cf. [16, 8.9(5)]):

$$
\begin{aligned}
& \int_{0}^{\infty} t^{\alpha+1 / 2} e^{-1 / 2 t^{2}} L_{n}^{\alpha}\left(t^{2}\right) e^{1 / 2 i(\operatorname{cotg} \gamma)\left(t^{2}+\lambda^{2}\right)} J_{\alpha}\left(\frac{\lambda t}{\sin 1 / 2 \gamma}\right) \frac{(\lambda t)^{1 / 2}}{\sin 1 / 2 \gamma} d t \\
& =(-1)^{n} e^{i(\pi-\gamma)(n+1 / 2 \alpha+1 / 2)} \lambda^{\alpha+1 / 2} e^{-1 / 2 \lambda^{2}} L_{n}^{\alpha}\left(\lambda^{2}\right) .
\end{aligned}
$$

Consider now the matrix elements $\left(D_{k}^{+}(g) v_{k, \lambda^{\prime}}^{L}, v_{k, \lambda}^{L}\right)$ and mixed basis matrix elements $\left(D_{k}^{+}(g) v_{k, \lambda^{\prime}}^{L^{\prime}}, v_{k, \lambda}^{L}\right)$, where $L, L^{\prime}$ are two of the subgroups $N, H, K$ and where $k$ and $g$ are held fixed as parameters, while the orthogonality is considered in the labels $\lambda, \lambda^{\prime}$ of the subgroup representations (cf. cases IIa and IIb of §6). In Table 10 below we summarize which orthogonal special functions arise in this way.

Let us quote these results more explicitly from the paper by Basu and Wolf. In each of the following formulas $g$ denotes the matrix element $\left(\begin{array}{ll}a & b \\ c & d\end{array}\right)$ of $G$.

$N-N$ matrix elements: Bessel functions:

$\left(D_{k}^{+}(g) v_{k, \rho^{\prime}}^{N}, v_{k, \rho}^{N}=e^{-i \pi k} b^{-1}\left(\rho \rho^{\prime}\right)^{1 / 2} e^{1 / 2 i b^{-1}\left(d \rho^{2}+a \rho^{\prime 2}\right)} J_{2 k-1}\left(b^{-1} \rho \rho^{\prime}\right), \quad b \neq 0\right.$. 


\begin{tabular}{c|c|c|c} 
& $D_{k}^{+}(g) v_{k, \rho^{\prime}}^{N}$ & $D_{k}^{+}(g) v_{k, \mu^{\prime}}^{H}$ & $D_{k}^{+}(g) v_{k, m^{\prime}}^{K}$ \\
\hline$v_{k, \rho}^{N}$ & Bessel functions & $\begin{array}{c}\text { Laguerre functions } \\
\text { (Mellin if } g=e)\end{array}$ & $\begin{array}{c}\text { Laguerre } \\
\text { polynomials }\end{array}$ \\
\hline$v_{k, \mu}^{H}$ & & $\begin{array}{c}\text { Meixner-Pollaczek } \\
\text { functions }\end{array}$ & $\begin{array}{c}\text { Meixner-Pollaczek } \\
\text { polynomials }\end{array}$ \\
\hline$v_{k, m}^{K}$ & & & Meixner polynomials
\end{tabular}

Table 10

This follows immediately from (7.14) and (7.1). The corresponding integral transform is essentially the Hankel transform.

$H-H$ matrix elements: Meixner-Pollaczek functions:

$\left(D_{k}^{+}(g) v_{k, \mu^{\prime}}^{H}, v_{k, \mu}^{H}\right)=$ elementary factors $\times_{2} F_{1}\left(k-i \mu, k+i \mu^{\prime} ; 2 k ;(a d)^{-1}\right)$.

We call the right hand side, in its dependence on $\mu$ or $\mu^{\prime}$, a Meixner-Pollaczek function because it bears the same relationship to Meixner-Pollaczek polynomials (cf. (5.8)) as Jacobi functions do to Jacobi polynomials (cf. (4.7),(4.6)). The corresponding unitary integral transform does not seem to be widely known in literature.

$K-K$ matrix elements: Meixner polynomials:

$\left(D_{k}^{+}(g) v_{k, m^{\prime}}^{K}, v_{k, m}^{K}\right)=$ elementary factors $\times M_{m-k}\left(m^{\prime}-k, 2 k ; \frac{a^{2}+b^{2}+c^{2}+d^{2}-2}{a^{2}+b^{2}+c^{2}+d^{2}+2}\right)$.

$N$ - $H$ mixed basis matrix elements: Laguerre functions:

$\left(D_{k}^{+}(g) v_{k, \mu}^{H}, v_{k, \rho}^{N}\right)=$ elementary factors $\times{ }_{1} F_{1}\left(k+i \mu ; 2 k ;-(2 a b)^{-1} i \rho^{2}\right), \quad a, b \neq 0$.

Consider, for each $\mu \in \mathbb{R}$, the right hand side as a function of $\rho$. It bears the same relationship to Laguerre polynomials as Jacobi functions do to Jacobi polynomials. The limit case of (7.21) for $g \rightarrow e$ follows from (7.14) and (7.15):

$\left(v_{k, \mu}^{H}, v_{k, \rho}^{N}\right)=\pi^{-1 / 2} \rho^{-1 / 2+1 / 2 i \mu}$.

This is the integral kernel of the Mellin transform, which is, by a trivial change of variables, just the Fourier transform.

$N-K$ mixed basis matrix elements: Laguerre polynomials.

In view of the Iwasawa decomposition $G=N H K$, these matrix elements, in their dependence on $g$, are already determined when they are given for $g \in H$. As $H$ acts quite trivially on $v_{k, \rho}^{N}$ (in view of (7.4),(7.14)), it is sufficient to consider the matrix element at $g=e$. By (7.16) this equals

$\left(v_{k, m}^{K}, v_{k, \rho}^{N}\right)=v_{k, m}^{K}(\rho)=$ elementary factors $\times L_{m-k}^{2 k-1}\left(\rho^{2}\right)$.

$H-K$ mixed basis matrix elements: Meixner-Pollaczek polynomials:

$\left(D_{k}^{+}(g) v_{k, m}^{K}, v_{k, \mu}^{H}\right)=$ elementary factors $\times P_{m-k}^{(k)}\left(\mu ; 1 / 2 \arccos \left(\frac{(a c+b d)^{2}-1}{(a c+b d)^{2}+1}\right)\right)$.

Some remarks can be made at this stage. Of the six cases just discussed there are three orthogonal polynomial cases $(K-K, K-H$ and $K-N)$, in correspondence with the fact that $K$ is the only compact group among $K, H, N$. The matrix elements satisfy second order differential equations in the $\rho$-variable but second order difference equations in the $\mu$ - and $m$-variables, where the differences in the case of $\mu$ are taken in imaginary direction. In case of a second order differential equation (first row of Table 10), the spectral theory of differential equations with regular singularities can be used, but, in particular in the $H-H$ case, there is missing an analogous spectral theory of second order difference equations.

Both the conjugacy class of elliptic and of hyperbolic subgroups have $N$ in their closure:

$$
\left(\begin{array}{ll}
\lambda^{1 / 2} & 0 \\
0 & \lambda^{-1 / 2}
\end{array}\right]\left[\begin{array}{rr}
\cos (c \lambda) & -\sin (c \lambda) \\
\sin (c \lambda) & \cos (c \lambda)
\end{array}\right]\left[\begin{array}{ll}
\lambda^{1 / 2} & 0 \\
0 & \lambda^{-1 / 2}
\end{array}\right]^{-1}=\left(\begin{array}{rr}
\cos (c \lambda) & -\lambda \sin (c \lambda) \\
\lambda^{-1} \sin (c \lambda) & \cos (c \lambda)
\end{array}\right)
$$


and

$$
\left[\begin{array}{cc}
1 / 2 \sqrt{2} \lambda^{1 / 2} & -1 / 2 \sqrt{2} \lambda^{1 / 2} \\
1 / 2 \sqrt{2} \lambda^{-1 / 2} & 1 / 2 \sqrt{2} \lambda^{-1 / 2}
\end{array}\right]\left[\begin{array}{cc}
e^{\lambda c} & 0 \\
0 & e^{-\lambda c}
\end{array}\right]\left[\begin{array}{cc}
1 / 2 \sqrt{2} \lambda^{1 / 2} & -1 / 2 \sqrt{2} \lambda^{1 / 2} \\
1 / 2 \sqrt{2} \lambda^{-1 / 2} & 1 / 2 \sqrt{2} \lambda^{-1 / 2}
\end{array}\right]^{-1}=\left[\begin{array}{rr}
\operatorname{ch}(c \lambda) & \lambda \operatorname{sh}(c \lambda) \\
\lambda^{-1} \operatorname{sh}(c \lambda) & \operatorname{ch}(c \lambda)
\end{array}\right)
$$

tend both to $\left(\begin{array}{ll}1 & 0 \\ c & 1\end{array}\right)$ as $\lambda \downarrow 0$. There are corresponding limit transitions for the matrix elements of $D_{k}^{+}$with respect to the various subgroups. We display this in Table 11 below.

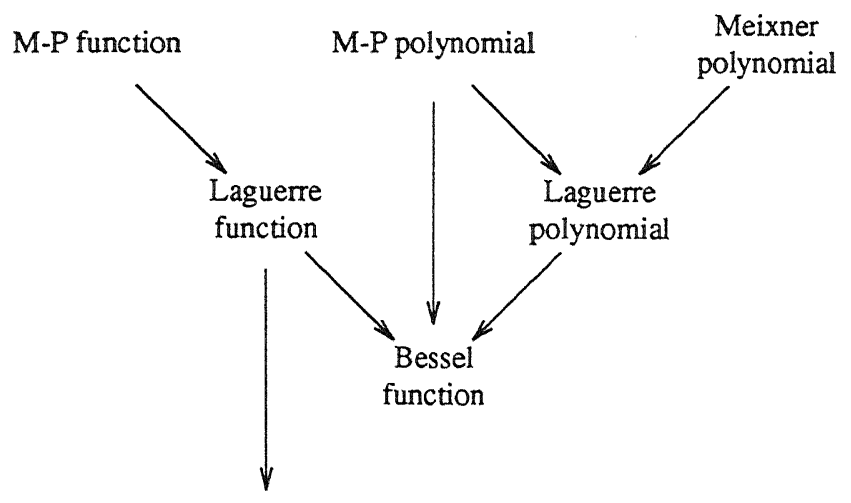

Mellin

Table 11

Of course, the various matrix elements encountered here offer ample illustration of formula (6.8). In particular, we obtain integral transforms sending Laguerre or Meixner-Pollaczek polynomials to polynomials of similar kind. Moreover, the various cases are connected by limit transitions, cf. Table 12 below.

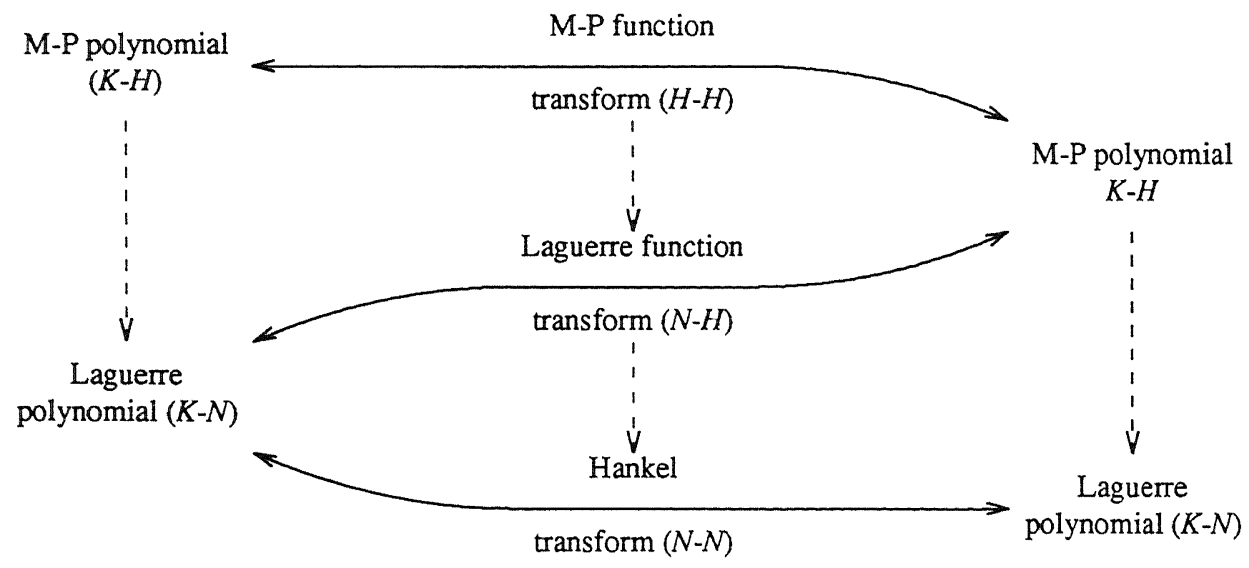

Table 12

By (7.22) the Laguerre function transform simplifies into the Mellin transform for $g=e$. That particular case in Table 12 then corresponds to the well-known formula

$$
\int_{0}^{\infty} \frac{n ! e^{-i n \phi}}{(2 a)_{n}} e^{-1 / 2 x(1+i \operatorname{cotg} \phi)} x^{a} L_{n}^{2 a-1}(x) x^{-1-i \lambda} d x
$$




$$
=e^{(i a-\lambda)(\phi-1 / 2 \pi)}(2 \sin \phi)^{a-i \lambda} \Gamma(a-i \lambda) P_{n}^{(a)}(\lambda ; \phi) .
$$

\section{Group theoretic interpretations of Hahn and Racah polynomials}

Hahn polynomials have a group theoretic interpretation as Clebsch-Gordan coefficients for $S U(2)$. We can understand these coefficients as overlap coefficients for bases with respect to two different subgroup reductions of $S U(2) \times S U(2)$. Let $T^{l}$ be the $(2 l+1)$-dimensional irreducible unitary representation of $S U(2)$ (cf. end of $\$ 6)$. Denote the (1-dimensional) irreducible representations of the subgroup $U(1)$ of $S U(2)$ by $\delta_{n}$ :

$$
\delta_{n}\left(\operatorname{diag}\left(e^{-1 / 2 i \theta}, e^{1 / 2 i \theta}\right)\right):=e^{i n \theta}, \quad n \in 1 / 2 \mathbb{Z} .
$$

For $S U(2) \times S U(2)$, together with its irreducible unitary representations $T^{l_{1}} \otimes T^{l_{2}}$, Table 13 below presents two subgroup reductions similar to Table 7.

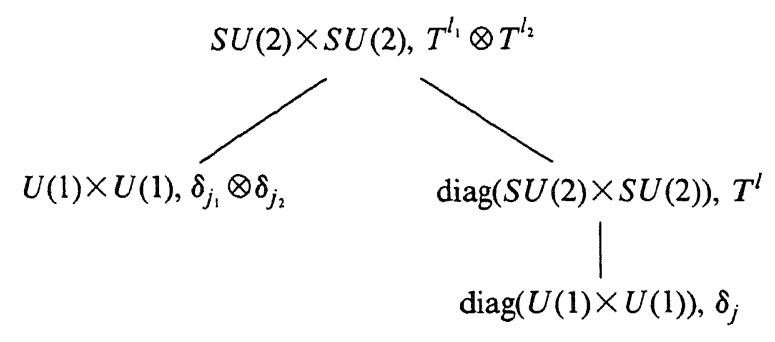

Table 13

There are corresponding orthonormal bases $\left\{v_{l_{1}, l_{2} ; j_{1}, j_{2}}\right\}$ and $\left\{w_{\left.l_{1}, l_{2} ; l_{j}\right\}}\right\}$ of $\mathcal{G C}\left(T^{l_{1}} \otimes T^{l_{2}}\right)$. Consider the overlap coefficients (cf. case IIb in §6)

$$
\psi^{l_{1}, l_{2}}\left(j_{1}, j_{2} ; l, j\right):=\left(w_{l_{1}, l_{2} ; l, j}, v_{l_{1}, l_{2} ; j_{1}, j_{2}}\right) \text {. }
$$

It turns out that these overlap coefficients vanish if $j \neq j_{1}+j_{2}$, so we can fix $j$ as a parameter, put $j_{2}:=j-j_{1}$ and only consider

$$
\psi^{l_{1}, l_{2}, j}\left(j_{1}, l\right):=\left(w_{l_{1}, l_{2}: l_{1}, j}, v_{l_{1}, l_{2} ; j_{1}, j-j_{1}}\right) .
$$

By construction, the left hand side yields the matrix coefficient of a unitary matrix. It is usually denoted by

$$
C_{j_{1}, j_{2}, j}^{l_{1}, l_{2}, l}:=\left(w_{l_{1}, l_{2} ; l, j}, v_{l_{1}, l_{2} ; j_{1}, j_{2}}\right), \quad j=j_{1}+j_{2},
$$

a so-called Clebsch-Gordan coefficient or (with slightly different notation) a $3 j$-symbol. It can be evaluated as

$$
\begin{gathered}
C_{j_{1}, j_{2}, j}^{l_{1}, l_{2}, l}=\text { elementary factors } \times{ }_{3} F_{2}\left(\begin{array}{c}
-l_{1}+l_{2}-l,-l_{1}+l_{2}+l+1,-l_{1}+j_{1} \\
-l_{1}+l_{2}+j+1,-2 l_{1}
\end{array} \mid 1\right), \\
l_{1}-l_{2} \leqslant j \leqslant l_{2}-l_{1} \leqslant l \leqslant l_{1}+l_{2} ;-l_{1} \leqslant j_{1} \leqslant l_{1} ; j_{1}+j_{2}=j .
\end{gathered}
$$

By symmetries the general case can be reduced to (8.3). It follows from (8.3) and (5.5) that we have obtained Hahn polynomials:

$$
C_{1 / 2 N-x, 1 / 2(\alpha-\beta), N)+x, 1 / 2(\alpha-\beta)}^{1 / 2,1 / 2(N+\alpha+\beta), 1 / 2(\alpha)}=\text { elementary factors } \times Q_{n}(x ; \alpha, \beta, N) .
$$

The row and column orthogonality of the Clebsch-Gordan matrix yield the orthogonality relations for Hahn and dual Hahn polynomials. See, for instance, Vilenkin [36, Ch.3] and Koornwinder [25] for a more detailed treatment of Clebsch-Gordan coefficients.

Racah polynomials (cf. (5.1)) were first obtained when it was recognized that the Racah coefficients for $S U(2)$ can be expressed in terms of orthogonal polynomials (see Wilson [37]). Racah 
coefficients are the overlap coefficients for certain subgroup reductions of $S U(2) \times S U(2) \times S U(2)$. Write

$$
\begin{aligned}
& G_{1} \times G_{2} \times G_{3}:=S U(2) \times S U(2) \times S U(2), \\
& G_{i, j}:=\operatorname{diag}\left(G_{i} \times G_{j}\right), \quad i \neq j, \\
& G_{0}:=\operatorname{diag}\left(G_{1} \times G_{2} \times G_{3}\right), \\
& K_{0}:=\operatorname{diag}(U(1) \times U(1) \times U(1)) .
\end{aligned}
$$

Use again the irreducible unitary representations $T^{l}$ of $S U(2)$ and $\delta_{j}$ of $U(1)$. Then we have three subgroup reductions for the irreducible unitary representations $T^{l_{1}} \otimes T^{l_{2}} \otimes T^{l_{3}}$ of $G_{1} \times G_{2} \times G_{3}$ as given in Table 14 below.

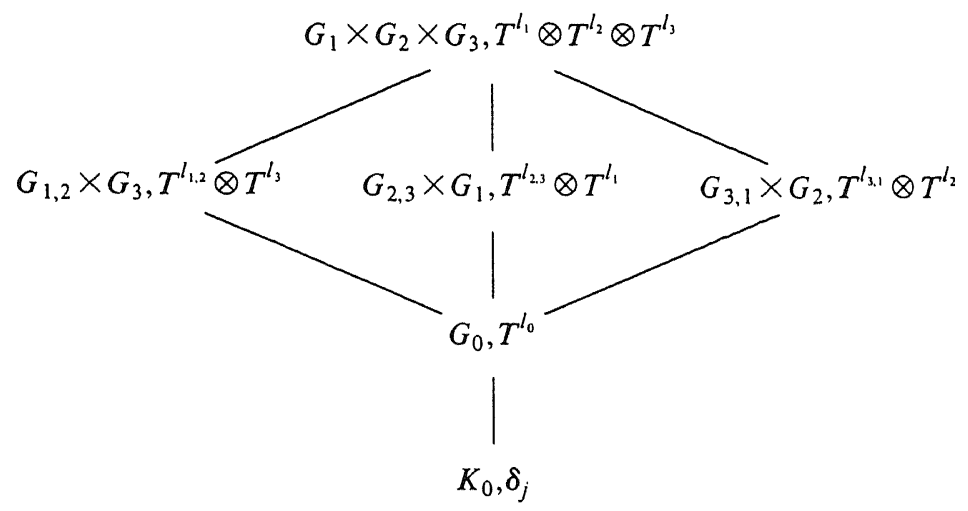

Table 14

There are three corresponding orthonormal bases $u_{l_{1}, l_{2}, l_{3} ; l_{1,2}, l_{0}, j}, v_{l_{1}, l_{2}, l_{3} ; l_{2,3}, l_{0}, j}, w_{l_{1}, l_{2}, l_{3} ; l_{3,1}, l_{0}, j}$. Now consider $l_{1}, l_{2}, l_{3}, l_{0}$ as parameters and define the Racah coefficients or 6j-symbols as the overlap coefficients

$$
R_{l_{1,2}, l_{1,3}}^{l_{1}, l_{2}, l_{3}, l_{0}}:=\left(u_{l_{1}, l_{2}, l_{3} ; l_{1,2}, l_{0}, j}, v_{l_{1}, l_{2}, l_{3} ; l_{2,3}, l_{0}, j}\right)
$$

These coefficients can be expressed in terms of ${ }_{4} F_{3}$-hypergeometric functions of argument 1 , and can next be written in terms of Racah polynomials (5.1). The row and column orthogonality of the Racah matrix yield the orthogonality relations for the Racah polynomials. See Biedenharn and Louck [10, Ch.3,§18] for the general theory of Racah coefficients and Wilson [37, §5] for a proof that these matrix coefficients can be expressed in terms of Racah polynomials.

There is a fourth subgroup reduction from $G_{1} \times G_{2} \times G_{3}, T^{l_{1}} \otimes T^{l_{2}} \otimes T^{l_{3}}$ to $K_{0}, \delta_{j}$ via the intermediate stage $K_{1} \times K_{2} \times K_{3}, \delta_{j_{1}}, \delta_{j_{2}}, \delta_{j_{3}}\left(K_{i}\right.$ being the subgroup $U(1)$ in $\left.G_{i}\right)$. This determines an orthonormal basis $\left\{e_{l_{1}, j_{1}} \otimes e_{l_{2}, j_{2}} \otimes e_{l_{3}, j_{3}}\right\}\left(j_{1}+j_{2}+j_{3}=j\right)$ for the representation space and we have

$$
\begin{array}{r}
R_{l_{1}, 2, l_{1}, 3}^{l_{1}, l_{2}, l_{1}, l_{0}}=\sum_{j_{1}, j_{2}, j_{3}}\left(u_{l_{1}, l_{2}, l_{3} ; l_{1,2}, l_{0}, j}, e_{l_{1}, j_{1}} \otimes e_{l_{2}, j_{2}} \otimes e_{l_{3}, j_{3}}\right) \\
\times\left(e_{l_{1}, j_{1}} \otimes e_{l_{2}, j_{2}} \otimes e_{l_{3}, j_{3}}, v_{l_{1}, l_{2}, l_{3} ; l_{2,3}, l_{0}, j}\right)
\end{array}
$$

(in the spirit of (6.8)). The two overlap coefficients occurring as factors in the sum at the right hand side of (8.6) can be written as products of two Clebsch-Gordan coefficients and they must yield orthogonal systems. These orthogonal systems can be written as functions depending on two discrete variables which are products of elementary factors and two Hahn polynomials. It would be interesting to see if these functions coincide with the orthogonal polynomials in two variables built from Hahn polynomials which are obtained by Dunkl [12, \$4] in connection with harmonic analysis on the symmetric group. Anyhow, (8.6) shows that the two orthogonal systems of functions in two variables can be expanded in terms of each other by means of Racah coefficients. We will shortly 
meet other examples of this phenomenon.

It is natural to expect similar group theoretic interpretations for continuous $\mathrm{Hahn}$ and dual Hahn polynomials and for Wilson polynomials. One might speculate about decompositions of twoand threefold tensor products of representations of $S L(2, \mathbb{R})$. As far as I know, it is still an open problem to find such interpretations.

However, there is another group theoretic interpretation of Racah polynomials for which we know how to imitate it in the case of Wilson polynomials. This interpretation was earlier given in $[28, \S 4]$ and Nikiforov, Suslov and Uvarov [31, Ch.5, §3]. Let $\operatorname{harm}(n, p)$ be the space of spherical harmonics of degree $n$ on the unit sphere $S^{p-1}$ in $\mathbb{R}^{p}$. Let $O(p)$ be the group of real $p \times p$ orthogonal matrices and let $\pi_{n}^{P}$ be the natural irreducible unitary representation of $O(p)$ on harm $(n, p)$. Consider in this way the representation $\pi_{n}^{p+q+r}$ of $O(p+q+r)$. We have three subgroup reductions as given in Table 15 below.

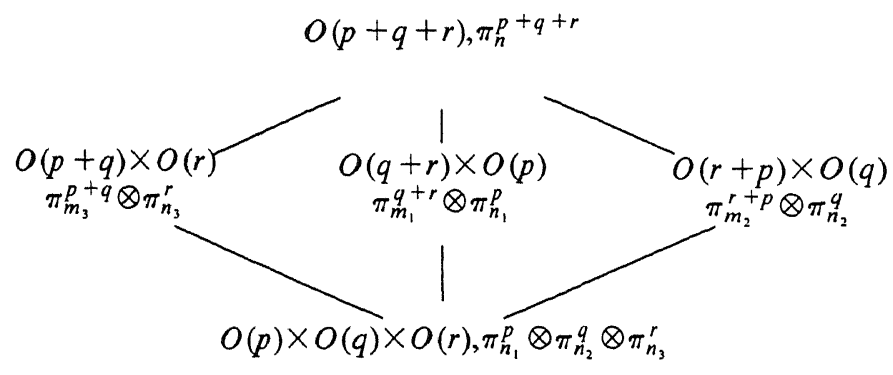

Table 15

Here

$n-m_{1}, n-m_{2}, n-m_{3}, m_{1}-n_{2}-n_{3}, m_{2}-n_{3}-n_{1}, m_{3}-n_{1}-n_{2}, n-n_{1}-n_{2}-n_{3} \in 2 \mathbb{Z}_{+}$.

Fix $n, n_{1}, n_{2}, n_{3}$ in Table 15. Corresponding to the three subgroup reductions there are three orthonormal bases for $\mathcal{H}\left(\pi_{n}^{p+q+r}\right)$ and the overlap coefficients turn out to be Racah coefficients again. This can be shown by realizing $\pi_{n}^{p+q+r}$ as a representation on harm $(n, p+q+r)$ and then giving explicit expressions for the three bases in terms of products of Jacobi polynomials. functions

In fact, by iteration of [23, Theorem 4.2], we obtain that harm $(n, p+q+r)$ is spanned by the

$$
\mathbb{R}^{p} \times \mathbb{R}^{q} \times \mathbb{R}^{r} \supset S^{p+q+r-1} \ni(x, y, z) \mapsto f_{n, m_{3}}^{n_{1}, n_{2}, n_{3}}(x, y, z) Y_{n_{1}}^{p}(x) Y_{n_{2}}^{q}(y) Y_{n_{3}}^{r}(z),
$$

where $n_{1}, n_{2}, n_{3}$ satisfy the inequalities (8.7), $Y_{n_{1}}^{p}, Y_{n_{2}}^{q}, Y_{n_{3}}^{r}$ are harmonic homogeneous polynomials of degree $n_{1}, n_{2}, n_{3}$, respectively, and

$$
\begin{aligned}
& f_{n_{1}, m_{3}}^{n_{1}, n_{2}, n_{3}}(x, y, z):=\left(|x|^{2}+|y|^{2}\right)^{1 / 2\left(m_{3}-n_{1}-n_{2}\right)} P_{1 / 2\left(m_{3}-n_{1}-n_{2}\right)}^{\left(1 / 2 q-1+n_{2}, 1 / p-1+n_{1}\right)}\left(1-2 \frac{|y|^{2}}{|x|^{2}+|y|^{2}}\right) \\
& \times P_{1 / 2\left(n-m_{3}-n_{3}\right)}^{\left(1 / 2 r-1+n_{3}, 1 /(p+q)-1+m_{3}\right)}\left(1-2|z|^{2}\right), \quad x \in \mathbb{R}^{p}, y \in \mathbb{R}^{q}, z \in \mathbb{R}^{r},|x|^{2}+|y|^{2}+|z|^{2}=1,
\end{aligned}
$$

with $P_{n}^{(\alpha, \beta)}$ being a Jacobi polynomial. By cyclic permutation in (8.8),(8.9) we get, in correspondence with Table 15, two other families $\left\{g_{n, m_{1}}^{n_{1}, n_{2}, n_{3}} Y_{n_{1}}^{p} Y_{n_{2}}^{q} Y_{n_{3}}^{r}\right\}$ and $\left\{h_{n, m_{2}}^{n_{1}, n_{2}, n_{3}} Y_{n_{1}}^{p_{1}} Y_{n_{2}}^{q} Y_{n_{3}}^{r}\right\}$ of functions which span harm $(n, p+q+r)$.

Fix $n_{1}, n_{2}, n_{3}, Y_{n_{1}}^{p_{1}}, Y_{n_{2}}^{q}, Y_{n_{3}}^{r}$ and write

$$
\begin{aligned}
& u:=|z|^{2}, v:=|y|^{2}, \nu:=1 / 2\left(n-n_{1}-n_{2}-n_{3}\right), \mu:=1 / 2\left(m_{3}-n_{1}-n_{2}\right), \\
& \mu^{\prime}:=1 / 2\left(m_{2}-n_{3}-n_{1}\right), \alpha:=1 / 2 r-1+n_{3}, \beta:=1 / 2 q-1+n_{2}, \gamma:=1 / 2 p-1+n_{1} .
\end{aligned}
$$

Then $f_{n, m_{3}}^{n_{1}, n_{2}, n_{3}}(x, y, z)$ can be written as 


$$
P_{\nu, \mu}^{\alpha, \beta, \gamma}(u, v):=P_{\nu-\mu}^{(\alpha, \beta+\gamma+2 \mu+1)}(1-2 u)(1-u)^{\mu} P_{\mu}^{(\beta, \gamma)}\left(1-2 \frac{v}{1-u}\right)
$$

and $h_{n, m_{2}}^{n_{1}, n_{2}, n_{3}}(x, y, z)$ as

$$
Q_{\nu, \mu^{\prime}}^{\alpha, \beta}(u, v):=P_{\nu-\mu^{\prime}}^{\left(\beta, \gamma+\alpha+2 \mu^{\prime}+1\right)}(1-2 v)(1-v)^{\mu^{\prime}} P_{\mu^{\prime}}^{(\gamma, \alpha)}\left(1-2 \frac{1-u-v}{1-v}\right) .
$$

Computation of the overlap coefficients now amounts to expanding $Q_{\nu, \mu^{\prime}}^{\alpha, \beta}$ in terms of the functions $P_{\nu, \mu}^{\alpha, \beta, \gamma}$.

Observe that, for arbitrary $\alpha, \beta, \gamma>-1$, both $\left\{P_{n, m}^{\alpha, \beta, \gamma}\right\}_{m}=0,1, \ldots, n$ and $\left\{Q_{n, m}^{\alpha, \beta, \gamma}\right\}_{m}=0,1, \ldots, n$ form an orthogonal basis of the space of all orthogonal polynomials of degree $n$ on the triangular region $\left\{(u, v) \in \mathbb{R}^{2} \mid u, v, 1-u-v>0\right\}$ with respect to the measure $u^{\alpha} v^{\beta}(1-u-v)^{\gamma} d u d v$. The two families are obtained by choosing two different ways of orthogonalizing monomials. (In fact, there are three such families, reflecting the symmetry of order three of the triangle.)

Thus, for arbitrary $\alpha, \beta, \gamma>-1$ there is an expansion

$$
Q_{\nu, \mu^{\prime}}^{\alpha, \beta, \gamma}(u, \nu)=\sum_{\mu=0}^{\nu} c_{\nu, \mu, \mu}^{\alpha, \beta, \gamma} P_{\nu, \mu}^{\alpha, \beta, \gamma}(u, v)
$$

The expansion coefficients are then obtained by elementary computations after putting $u:=0$ in (8.12). The result is

$c_{\nu, \mu, \mu}^{\alpha, \beta, \gamma}:=$ elementary factors $\times R_{\mu}\left(\mu^{\prime}\left(\mu^{\prime}+\alpha+\gamma+1\right) ; \gamma, \beta,-\nu-1, \alpha+\gamma+\nu+1\right)$,

where $R_{\mu}$ is a Racah polynomial. This result was first obtained by Dunkl [13, Theorem 1.7] as a limit case of a similar formula for Hahn polynomials in two variables.

Following the limit transitions in the Askey scheme (Table 3) we can take limits in (8.12) and (8.13). Thus we obtain that the two orthogonal bases

$$
(u, v) \mapsto L_{n-k}^{\alpha}(u) L_{k}^{\beta}(v)
$$

and

$$
(u, v) \mapsto L_{n-m}^{\alpha+\beta+2 m+1}(u+v)(u+v)^{m} P_{m}^{(\alpha, \beta)}\left(1-2 \frac{u}{u+v}\right)
$$

for orthogonal polynomials of degree $n$ on $\mathbb{R}_{+} \times \mathbb{R}_{+}$with respect to the measure $u^{\alpha} v^{\beta} e^{-u-v} d u d v$ can be expanded in terms of each other with Hahn polynomials as coefficients. This was obtained by Suslov [33] in connection with the Schrödinger equation for the Coulomb prcblem.

There are similar expansions for two orthogonal bases of the orthogonal polynomials on the unit disk with respect to the measure $\left(1-x^{2}-y^{2}\right)^{\alpha} d x d y$ (using symmetric Hahn polynomials) and for two orthogonal bases of the orthogonal polynomials on $\mathbb{R}^{2}$ with respect to the measure $\exp \left(-x^{2}-y^{2}\right) d x d y$ (using symmetric Krawtchouk polynomials).

\section{Group theoretic interpretation of Wilson polynomials}

The group theoretic interpretation of Racah polynomials related to Table 15 in $\$ 8$ admits a (formal) analytic continuation such that it becomes a group theoretic interpretation of Wilson polynomials. I will now summarize the results of $[28, \S 5]$, where this interpretation was given.

Let $\operatorname{Hyp}(p, q):=\left\{(x, y) \in \mathbb{R}^{p} \times\left.\mathbb{R}^{q}|-| x\right|^{2}+|y|^{2}=1\right\}$, an hyperboloid, and denote by $\operatorname{harm}(\lambda ; p, q)$ the class of hyperboloid harmonics of degree $i \lambda-1 / 2(p+q)+1$, i.e. the class of functions on $\operatorname{Hyp}(p, q)$ which are restrictions of $C^{\infty}$-functions on $\left\{(x, y) \in \mathbb{R}^{p} \times\left.\mathbb{R}^{q}|-| x\right|^{2}+|y|^{2}>0\right\}$ that are even, homogeneous of degree $i \lambda-1 / 2(p+q)+1$ and annihilated by the differential operator

$$
\frac{\partial^{2}}{\partial x_{1}^{2}}+\cdots+\frac{\partial^{2}}{\partial x_{p}^{2}}-\frac{\partial^{2}}{\partial y_{1}^{2}}-\cdots-\frac{\partial^{2}}{\partial y_{q}^{2}} .
$$

Let $O(p, q)$ be the group of linear transformations of $\mathbb{R}^{p+q}$ which leave the form $-|x|^{2}+|y|^{2}$ on $\mathbb{R}^{p} \times \mathbb{R}^{q}$ invariant. Then $O(p, q)$ naturally acts on $\operatorname{harm}(\lambda ; p, q)$. If $\lambda>0$ then we can associate an irreducible unitary representation $\tau_{\lambda}^{p, q}$ of $O(p, q)$ with this action (cf. Faraut [17]). 
Consider in this way the representation $\tau_{\lambda}^{p+q, r}$ of $O(p+q, r)$ on harm $(\lambda ; p+q, r)$. By formal analytic continuation of Table 15 one is led to three remarkable subgroup reductions of this representation. See Table 16 below.

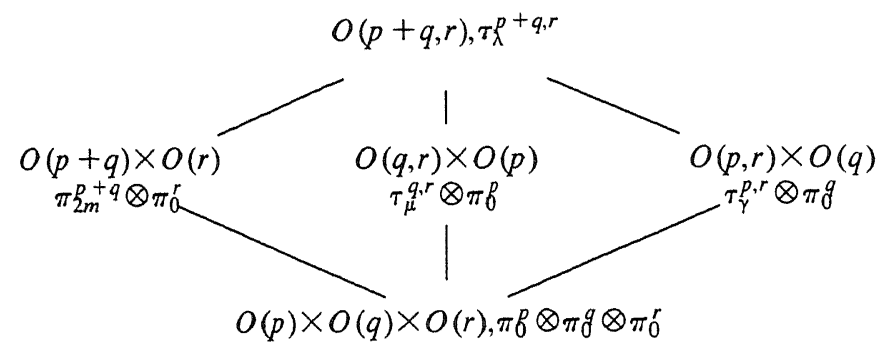

Table 16

For simplicity we have restricted ourselves to the case that the representation of the subgroup $O(p) \times O(q) \times O(r)$ at the bottom of Table 16 is trivial. (The representations $\pi$ in Table 16 are representations on spaces of spherical harmonics as in $\S 8$.)

Now fix $\lambda>0$ and $p, q, r$ in Table 16. The three subgroup chains determine three different (generalized) orthonormal bases for the $O(p) \times O(q) \times O(r)$-invariant elements in harm $(\lambda ; p+q, r)$. These basis elements have explicit expressions which are analytic continuations of (8.8),(8.9) (with the $Y$-factors trivial). In fact, the basis functions $\phi_{\lambda, m}\left(m \in \mathbb{Z}_{+}\right)$with respect to the first subgroup chain and $\psi_{\lambda, \mu}\left(\mu \in \mathbb{R}_{+}\right)$with respect to the second subgroup chain are given, up to a constant factor, by

$$
\begin{aligned}
\phi_{\lambda, m}(u, v) & :=\phi_{\lambda}^{(1 / 2(p+q)+2 m-1,1 / 2 r-1)}\left(\operatorname{arcsh}(u+v)^{1 / 2}\right)(u+v)^{m} P_{m}^{(1 / 2 p-1,1 / 2 q-1)}\left(\frac{-u+v}{u+v}\right), \\
& \psi_{\lambda, \mu}(u, v):=\phi_{\lambda}^{(1 / 2 p-1, i \mu)}\left(\operatorname{arcsh} u^{1 / 2}\right)(u+1)^{1 / 2 i \mu-1 / 4(q+r)+1 / 2} \\
& \times \phi_{\mu}(1 / 2 q-1,1 / 2 r-1)\left(\operatorname{arcsh} \frac{v^{1 / 2}}{(u+1)^{1 / 2}}\right),
\end{aligned}
$$

where $(x, y, z) \in \operatorname{Hyp}(p+q, r) \subset \mathbb{R}^{p} \times \mathbb{R}^{q} \times \mathbb{R}^{r}$ and $u:=|x|^{2}, v:=|y|^{2}$. The functions $P_{n}^{(\alpha, \beta)}$ and $\phi_{\lambda}^{(\alpha, \beta)}$ are Jacobi polynomials (4.6) and Jacobi functions (4.7), respectively.

The systems $\left\{\phi_{\lambda, m}\right\}_{\lambda \in \mathbf{R}_{+}, m \in \mathbf{Z}_{+}}$and $\left\{\psi_{\lambda, \mu}\right\}_{\lambda, \mu \in \mathbf{R}_{+}}$can be viewed as generalized orthogonal bases for $L^{2}$ on $\left(\mathbb{R}_{+}\right)^{2}$ with respect to the measure

$$
u^{1 / 2 p-1} v^{1 / 2 q-1}(1+u+v)^{1 / 2 r-1} d u d v \text {. }
$$

They are built as a kind of semidirect products from two orthogonal systems in one variable, just as the orthogonal polynomials (8.10),(8.11) on the triangle are built from Jacobi polynomials. This reduction to the one-variable case also makes it easy to invert the integral transform with $(9.1),(9.2)$ as kernel. In (9.2) one of the contributing one-variable transforms is the Jacobi function transform (4.12) with $\beta$ imaginary. This case of the Jacobi function transform seemed to be unobserved before it occurred here. mials:

The overlap coefficients between the systems in (9.1) and (9.2) can be given by Wilson polyno-

$$
\begin{aligned}
& \psi_{\lambda, \mu}=\sum_{m=0}^{\infty} \text { elementary factors } \\
& \times W_{m}\left(1 / 4 \mu^{2} ; \frac{p+2 i \lambda}{4}, \frac{p-2 i \lambda}{4}, \frac{q+r-2}{4}, \frac{q-r+2}{4}\right) \phi_{\lambda, m},
\end{aligned}
$$

with weak convergence, in analogy with (8.12) and (8.13). Essentially, the proof is also similar to that of (8.13), but much more complicated. Instead of restricting the arguments in (9.3) to one of 
the boundary lines of $\left(\mathbb{R}_{+}\right)^{2}$, one has to restrict arguments to a boundary line of $\left(\mathbb{R}_{+}\right)^{2}$ at infinity, i.e., one has to study the asymptotics of $(9.3)$ for $(u, v)$ large. In this way the identity $(4.16)=(4.17)$ can be interpreted as an asymptotic boundary case of (9.3) and the proof of (9.3) can be reduced to this identity.

\section{Remarks.}

1. The case $p=q=1$ of (9.3) and its group theoretic interpretation were earlier obtained by Boyer and Ardalan [11].

2. One remarkable difference of (9.3) with (8.12) is that (8.12) restricted to $u=0$ does not have an interpretation as a unitary transform between two orthogonal bases for $L^{2}$-spaces.

3. There is a limit case of $\left(9.100,(9.2),(9.3)\right.$ in which $\psi_{\lambda, \mu}$ tends to a product of two Laguerre functions (cf. (7.21)) and $\phi_{\lambda, m}$ to a product of a Laguerre function and a Jacobi polynomial. The Wilson polynomial in (9.3) tends to a continuous Hahn polynomial. See Suslov [33].

4. There is a challenging problem left by Table 16: What are the overlap coefficients relating the second and the third subgroup chain in Table 16 to each other? They might be naturally called Wilson functions, but what are they like? Maybe of ${ }_{4} F_{3}$-type, but probably of an even higher hypergeometric type. One may expect that everything in this paper can be obtained as a limit case of these yet virtual Wilson functions.

\section{References}

1. G.E. Andrews and R. Askey, "Classical orthogonal polynomials," pp. 36-62 in Polynômes Orthogonaux et Applications, ed. C. Brezinski, A. Draux, A.P. Magnus, P. Maroni, A. Ronveaux, Lecture Notes in Math. 1171, Springer (1985).

2. R. Askey, "Continuous Hahn polynomials," J. Phys. A: Math. Gen. 18, pp. L1017-L1019 (1985).

3. R. Askey and J. Wilson, "A set of hypergeometric orthogonal polynomials," SIAM J. Math. Anal. 13, pp. 651-655 (1982).

4. R. Askey and J. Wilson, Some basic hypergeometric orthogonal polynomials that generalize Jacobi polynomials, Memoirs Amer. Math. Soc. 319 (1985).

5. N.M. Atakishiyev and S.K. Suslov, "The Hahn and Meixner polynomials of an imaginary argument and some of their applications," J.Phys. A: Math. Gen. 18, pp. 1583-1596 (1985).

6,7,8. E. Badertscher and T.H. Koornwinder, "Continuous Hahn polynomials of argument $i d / d t$ and analysis on Riemannian symmetric spaces of constant curvature," CWI preprint, to appear.

9. D. Basu and K.B. Wolf, "The unitary irreducible representations of $\mathrm{SL}(2, \mathrm{R})$ in all subgroup reductions," J. Math. Phys. 23, pp. 189-205 (1982).

10. L.C. Biedenharn and J.D. Louck, Angular momentum in quantum physics, Encyclopedia of Math., Vol. 8, Addison-Wesley (1981).

11. C.P. Boyer and F. Ardalan, "On the decomposition $S O(p, 1) \supset S O(p-1,1)$ for most degenerate representations," J. Math. Phys. 12, pp. 2070-2075 (1971).

12. C.F. Dunkl, "A difference equation and Hahn polynomials in two variables," Pac. J. Math. 92, pp. $57-71$ (1981).

13. C.F. Dunkl, "Orthogonal polynomials with symmetry of order three," Can. J. Math. 36, pp. 685-717 (1984).

14. A. Erdélyi, W. Magnus, F. Oberhettinger, and F.G. Tricomi, Higher transcendental functions, Vols. I,II,III, McGraw-Hill $(1953,1953,1955)$.

15,16. A. Erdélyi, W. Magnus, F. Oberhettinger, and F.G. Tricomi, Tables of integral transforms, Vols. I,II, McGraw-Hill (1954).

17. J. Faraut, "Distributions sphériques sur les espaces hyperboliques," J. Math. Pures Appl. 58, pp. 369-444 (1979). 
18. J. Faraut, "Analyse harmonique sur les paires de Guelfand et les espaces hyperboliques," pp. 315-446 in Analyse harmonique, CIMPA, Nice (1982).

19. M. Flensted-Jensen, "Spherical functions on a simply connected semisimple Lie group. II. The Paley-Wiener theorem for the rank one case," Math. Ann. 228, pp. 65-92 (1977).

20. S. Helgason, "A duality for symmetric spaces with applications to group representations, II. Differential equations and eigenspace representations," Advances in Math. 22, pp. 187-219 (1976).

21. S. Helgason, Groups and geometric analysis, Academic Press (1984).

22. S. Karlin and J.L. McGregor, "The Hahn polynomials, formulas and an application," Scripta Math. 26, pp. 33-46 (1963).

23. T.H. Koornwinder, "The addition formula for Jacobi polynomials and spherical harmonics," SIAM J. Appl. Math. 25, pp. 236-246 (1973).

24. T.H. Koornwinder, "A new proof of a Paley-Wiener type theorem for the Jacobi transform," Ark. Mat. 13, pp. 145-159 (1975).

25. T.H. Koornwinder, "Clebsch-Gordan coefficients for $S U(2)$ and Hahn polynomials," Nieuw Archief voor Wiskunde (3) 29, pp. 140-155 (1981).

26. T.H. Koornwinder, "Krawtchouk polynomials, a unification of two different group theoretic interpretations," SIAM J. Math. Anal. 13, pp. 1011-1023 (1982).

27. T.H. Koornwinder, "Jacobi functions and analysis on noncompact semisimple Lie groups," pp. 1-85 in Special functions: Group theoretical aspects and applications, (R.A. Askey, T.H. Koornwinder, W. Schempp, eds.), Reidel (1984).

28. T.H. Koornwinder, "A group theoretic interpretation of Wilson polynomials," Report PMR8504, Centre for Math. and Computer Science, Amsterdam (1985).

29. T.H. Koornwinder, "Special orthogonal polynomial systems mapped onto each other by the Fourier-Jacobi transform," pp. 174-183 in Polynômes Orthogonaux et Applications, (C. Brezinski, A. Draux, A.P. Magnus, P. Maroni, A. Ronveaux, eds.), Lecture Notes in Math. 1171, Springer (1985).

30. J. Labelle, "Tableau d'Askey," pp. xxxvi-xxxvii in Polynômes Orthogonaux et Applications, ed. C. Brezinski, A. Draux, A.P. Magnus, P. Maroni, A. Ronveaux, Lecture Notes in Math. 1171, Springer (1985).

31. A.F. Nikiforov, S.K. Suslov, and V.B. Uvarov, Classical orthogonal poynomials of a discrete variable (Russian), Nauka, Moscow (1985).

32. S.N.M. Ruijsenaars, "Complete integrability of relativistic Calogero-Moser systems and elliptic function identities," preprint (1986).

33. S.K. Suslov, "The Hahn polynomials in the Coulomb problem (Russian)," Yadernaya Fiz. 40, no.1, pp. 126-132 (1984).

34. G. Szegö, Orthogonal polynomials, American Math. Society Colloquium Publications, Vol. 23, Fourth ed. (1975).

35. K. Trimèche, "Transformation intégrale de Weyl et théorème de Paley-Wiener associés à un

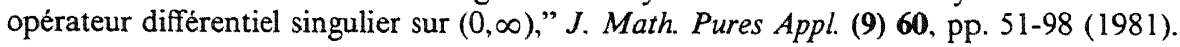

36. N.Ja. Vilenkin, Special functions and the theory of group representations, Amer. Math. Soc. Transl. of Math. Monographs, Vol. 22 (1968).

37. J.A. Wilson, "Some hypergeometric orthogonal polynomials," SIAM J. Math. Anal. 11, pp. 690-701 (1980). 